\title{
Exotic decays of a heavy neutral Higgs through HZ/AZ channel
}

\author{
Baradhwaj Coleppa, Felix Kling and Shufang Su \\ Department of Physics, University of Arizona, \\ 1118 E. 4th st., P.O. Box 210081, Tucson, AZ 85721, U.S.A. \\ E-mail: baradhwa@email.arizona.edu, kling@email.arizona.edu, \\ shufang@email.arizona.edu
}

ABSTRACT: Models of electroweak symmetry breaking with extended Higgs sectors are theoretically well motivated. In this study, we focus on the Two Higgs Doublet Model with a low energy spectrum containing scalars $H$ and a pseudoscalar $A$. We study the decays $A \rightarrow H Z$ or $H \rightarrow A Z$, which could reach sizable branching fractions in certain parameter regions. With detailed collider analysis, we obtain model independent exclusion bounds as well as discovery reach at the $14 \mathrm{TeV}$ LHC for the process: $g g \rightarrow A / H \rightarrow H Z / A Z$, looking at final states $b b \ell \ell, \tau \tau \ell \ell$ and $Z Z Z(4 \ell+2 j)$ for $\ell=e, \mu$. We further interpret these bounds in the context of the Type II Two Higgs Doublet Model, considering three different classes of processes: $A \rightarrow h^{0} Z, A \rightarrow H^{0} Z$, and $H^{0} \rightarrow A Z$, in which $h^{0}$ and $H^{0}$ are the light and heavy CP-even Higgses respectively. For $100 \mathrm{fb}^{-1}$ integrated luminosity at the $14 \mathrm{TeV} \mathrm{LHC}$, we find that for parent particle mass around $300-400 \mathrm{GeV}, A \rightarrow h^{0} Z$ has the greatest reach when $H^{0}$ is interpreted as the $126 \mathrm{GeV}$ Higgs: most regions in the $\tan \beta$ versus $\sin (\beta-\alpha)$ plane can be excluded and a significant fraction at small and large $\tan \beta$ can be covered by discovery. For $126 \mathrm{GeV} h^{0}$, only relatively small $\tan \beta \lesssim 10(5)$ can be reached by exclusion (discovery) while a wide range of $\sin (\beta-\alpha)$ is accessible. For $A \rightarrow H^{0} Z$, the reach is typically restricted to $\sin (\beta-\alpha) \sim \pm 1$ with $\tan \beta \lesssim 10$ in bbll and $\tau \tau \ell \ell$ channels. The $Z Z Z(4 \ell 2 j)$ channel, on the other hand, covers a wide range of $0.3<|\sin (\beta-\alpha)|<1$ for $\tan \beta \lesssim 4$. $H^{0} \rightarrow A Z$ typically favors negative values of $\sin (\beta-\alpha)$, with exclusion/discovery reach possibly extending to all values of $\tan \beta$. A study of exotic decays of extra Higgses appearing in extensions of the Standard Model would extend the reach at the LHC and provides nice complementarity to conventional Higgs search channels.

Keywords: Higgs Physics, Beyond Standard Model

ARXIV EPRINT: 1404.1922 


\section{Contents}

1 Introduction 1

2 Scenarios with large $H \rightarrow A Z$ or $A \rightarrow H Z$

3 Current experimental limits $\quad 4$

4 Collider analysis $\quad 6$

$4.1 \quad A / H \rightarrow H Z / A Z \rightarrow b b \ell \ell+6$

$4.2 \quad A / H \rightarrow H Z / A Z \rightarrow \tau \tau \ell \ell \quad 10$

$4.3 \quad A \rightarrow H Z \rightarrow Z Z Z \rightarrow 4 \ell+2 j \quad 13$

5 Implications for the Type II 2HDM $\quad 14$

$5.1 g g \rightarrow A \rightarrow h^{0} Z \quad 17$

$5.2 \quad g g \rightarrow A \rightarrow H^{0} Z \quad 21$

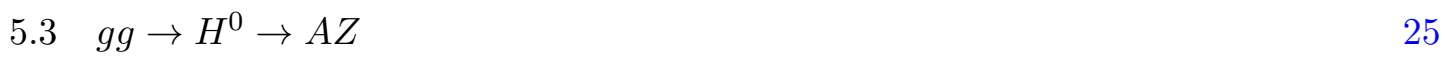

6 Conclusion 28

\section{Introduction}

The greatest experimental triumph of the Large Hadron Collider (LHC) till date is the discovery of a scalar resonance at $126 \mathrm{GeV}$ with properties consistent with that of the Standard Model (SM) Higgs [1-4]. The mass of this particle along with its spin [2, 4, 5] has now been established, and a complete characterization of all its possible decay modes is underway. At the same time, from the theoretical front, we have now known for a while that the SM, though in excellent agreement with experiments, has to be supplanted with other dynamics if it is to explain many puzzles facing particle physics today, viz., the hierarchy problem, neutrino masses, and the nature of dark matter, to name a few. Many beyond the SM scenarios are constructed to explain one or many of these puzzles, and are becoming more constrained by the Higgs observation at the LHC. This is particularly true for theories constructed with an extended Higgs sector. Well known examples are the Minimal Supersymmetric Standard Model (MSSM) [6-8], Next to Minimal Supersymmetric Standard Model (NMSSM) [9, 10] and Two Higgs Doublet Models (2HDM) [11-14]. In addition to the SM-like Higgs boson in these models, the low energy spectrum includes other CP-even Higgses, CP-odd Higgses, as well as charged ones.

Models with an extended Higgs sector hold a lot of phenomenological interest. The discovery of extra Higgses would be an unambiguous evidence for new physics beyond the SM. Other than the decay of these extra Higgses into the SM final states $\gamma \gamma, Z Z, W W$, $b b$ and $\tau \tau$, which have been the focus of the current Higgs searches, the decay of heavy 
Higgses into light Higgses, or Higgs plus gauge boson final states could also be sizable. Such decays are particularly relevant as the $126 \mathrm{GeV}$ resonance could show up as a decay of a heavier state, opening up the interesting possibility of using the SM-like Higgs to discover its heavier counterparts. It is thus timely to study these exotic Higgs decay channels and fully explore the experimental discovery potential for the enlarged Higgs sector.

In this paper, we focus on the decays $H \rightarrow A Z$ or $A \rightarrow H Z$, with $H$ and $A$ referring to generic CP-even and CP-odd Higgs, respectively. ${ }^{1}$ We consider leptonic decays of the $Z$, with the $A / H$ in the final states decaying to either a pair of fermions ( $b b$ or $\tau \tau$ ) or $Z Z$ and explore the exclusion bounds as well as discovery reach at the LHC for various combinations of $\left(m_{A}, m_{H}\right)$.

In the 2HDM or NMSSM, both decays $H_{i} \rightarrow A_{j} Z$ and $A_{i} \rightarrow H_{j} Z$ could appear with large branching fractions as shown in [15-18]. Ref. [19] also argued that $A \rightarrow h^{0} Z$ could have a sizable branching fraction in the low $\tan \beta$ region of the MSSM with the light CP-

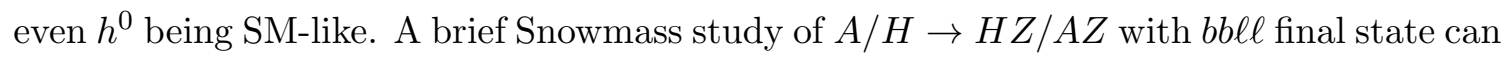
be found in ref. [20]. Another Snowmass study of heavy Higgses [21] explored sensitivities in the $H^{0} \rightarrow Z Z \rightarrow 4 \ell$ and $A \rightarrow Z h^{0} \rightarrow b b \ell \ell, \tau \tau \ell \ell$ channels at the $14 \mathrm{TeV}$ and $33 \mathrm{TeV}$ LHC, focusing on the case with $h^{0}$ being the $126 \mathrm{GeV}$ Higgs. In our study, we consider a variety of daughter Higgs masses in bbll and $\tau \tau \ell \ell$ channels, and analyze $A \rightarrow H^{0} Z \rightarrow Z Z Z$ in addition. We also interpret the search results in the context of the Type II 2HDM.

The paper is organized as follows. In section 2, we present a brief overview of models and parameter regions where the channels under consideration can be significant. In section 3, we summarize the current experimental search limits on heavy Higgses. In sec-

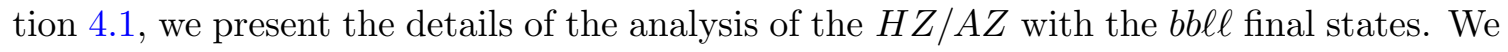
also show model-independent results of $95 \%$ C.L. exclusion as well as $5 \sigma$ discovery limits for $\sigma \times \mathrm{BR}(g g \rightarrow A / H \rightarrow H Z / A Z \rightarrow b b \ell \ell)$ at the $14 \mathrm{TeV}$ LHC with 100,300 and $1000 \mathrm{fb}^{-1}$ integrated luminosity. In sections 4.2 and 4.3, we present the analysis for the $\tau \tau \ell \ell$ and $Z Z Z$ final states, respectively. In section 5, we study the implications of the collider search limits on the parameter regions of the Type II 2HDM. We conclude in section 6.

\section{Scenarios with large $H \rightarrow A Z$ or $A \rightarrow H Z$}

In the 2HDM, we introduce two $\mathrm{SU}(2)$ doublets $\Phi_{i}, i=1,2$ :

$$
\Phi_{i}=\left(\begin{array}{c}
\phi_{i}^{+} \\
\left(v_{i}+\phi_{i}^{0}+i G_{i}\right) / \sqrt{2}
\end{array}\right),
$$

where $v_{1}$ and $v_{2}$ are the vacuum expectation values of the neutral components which satisfy the relation: $\sqrt{v_{1}^{2}+v_{2}^{2}}=246 \mathrm{GeV}$ after electroweak symmetry breaking. Assuming a discrete $\mathcal{Z}_{2}$ symmetry imposed on the Lagrangian, we are left with six free parameters, which can be chosen as four Higgs masses $\left(m_{h}, m_{H}, m_{A}, m_{H^{ \pm}}\right)$, the mixing angle $\alpha$ between the two CP-even Higgses, and the ratio of the two vacuum expectation values,

\footnotetext{
${ }^{1}$ Note that we use $h^{0}$ and $H^{0}$ to refer to the lighter or the heavier CP-even Higgs for models with two CP-even Higgs bosons. When there is no need to specify, we use $H$ to refer to the CP-even Higgses.
} 
$\tan \beta=v_{2} / v_{1}$. In the case in which a soft breaking of the $\mathcal{Z}_{2}$ symmetry is allowed, there is an additional parameter $m_{12}^{2}$.

The mass eigenstates contain a pair of CP-even Higgses: $h^{0}, H^{0}$, one CP-odd Higgs, $A$ and a pair of charged Higgses $H^{ \pm}:^{2}$

$$
\left(\begin{array}{c}
H^{0} \\
h^{0}
\end{array}\right)=\left(\begin{array}{cc}
\cos \alpha & \sin \alpha \\
-\sin \alpha & \cos \alpha
\end{array}\right)\left(\begin{array}{l}
\phi_{1}^{0} \\
\phi_{2}^{0}
\end{array}\right), \quad \begin{gathered}
A=-G_{1} \sin \beta+G_{2} \cos \beta \\
H^{ \pm}=-\phi_{1}^{ \pm} \sin \beta+\phi_{2}^{ \pm} \cos \beta .
\end{gathered}
$$

Two types of couplings that are of particular interest are $Z A H^{0} / h^{0}$ couplings and $H^{0} / h^{0} V V$ couplings, with $V$ being the SM gauge bosons $W^{ \pm}$and $Z$. Both are determined by the gauge coupling structure and the mixing angles. The couplings for $Z A H^{0}$ and $Z A h^{0}$ are [22]:

$$
g_{Z A H^{0}}=-\frac{g \sin (\beta-\alpha)}{2 \cos \theta_{w}}\left(p_{H^{0}}-p_{A}\right)_{\mu}, \quad g_{Z A h^{0}}=\frac{g \cos (\beta-\alpha)}{2 \cos \theta_{w}}\left(p_{h^{0}}-p_{A}\right)_{\mu},
$$

with $g$ being the $\mathrm{SU}(2)$ coupling, $\theta_{w}$ being the Weinberg angle and $p_{\mu}$ being the incoming momentum of the corresponding particle.

The $H^{0} V V$ and $h^{0} V V$ couplings are:

$$
g_{H^{0} V V}=\frac{m_{V}^{2}}{v} \cos (\beta-\alpha), \quad g_{h^{0} V V}=\frac{m_{V}^{2}}{v} \sin (\beta-\alpha) .
$$

Note that $A$ always couples to the non-SM-like Higgs more strongly. If we demand $h^{0}\left(H^{0}\right)$ to be SM-like, then $|\sin (\beta-\alpha)| \sim 1(|\cos (\beta-\alpha)| \sim 1)$ is preferred, and the $Z A H^{0}\left(Z A h^{0}\right)$ coupling is unsuppressed. Therefore, in the $h^{0}-126$ case, $A$ is more likely to decay to $H^{0} Z$ than $h^{0} Z$, unless the former decay is kinematically suppressed. $H^{0} \rightarrow A Z$ could also be dominant once it is kinematically open. Particularly for a heavy $H^{0}$, as we will demonstrate later in section $5, H^{0} \rightarrow A Z$ can have a large branching fraction in the $\sin (\beta-\alpha)= \pm 1$ regions. On the contrary, for $H^{0}$ being SM-like with $|\cos (\beta-\alpha)| \sim 1$, $A \rightarrow h^{0} Z$ dominates over $H^{0} Z$ channel. For very light $m_{A}, h^{0} \rightarrow A Z$ could also open. The detectability of this channel, however, is challenging given the soft or collinear final decay products from a light $A$. Therefore, for our discussion below, we will focus on the cases $A \rightarrow h^{0} Z, H^{0} Z$ and $H^{0} \rightarrow A Z$ only.

In the generic $2 \mathrm{HDM}$, there are no mass relations between the pseudoscalar and the scalar states. Thus, the decays $A \rightarrow h^{0} Z, H^{0} Z$ and $H^{0} \rightarrow A Z$ can happen in different regions of parameter spaces. It was shown in ref. [23] that in the Type II 2 HDM with $\mathcal{Z}_{2}$ symmetry, imposing all experimental and theoretical constraints still leaves sizable regions in the parameter space. In those parameter spaces, such exotic decays can have unsuppressed decay branching fractions. It was also pointed out in ref. [11] that in the Type I $2 \mathrm{HDM}$, for $\cos ^{2}(\alpha-\beta)>1 / 2$, the decay $h^{0} \rightarrow A Z$ will actually dominate the $W W$ decay for a light $A$. Results obtained in this study can also be applied to the CP-violating 2HDM in which $H_{i} \rightarrow H_{j} Z$ could be sizable with $H_{i, j}$ being mixtures of CP-even and CPodd states. Appropriate rescaling of the production cross sections and decay branching fractions is needed to recast the results.

\footnotetext{
${ }^{2}$ For more details about the model, see ref. [11].
} 
The Higgs sector in the MSSM is more restricted, given that the quartic Higgs couplings are fixed by the gauge couplings and the tree-level Higgs mass matrix only depends on $m_{A}$ and $\tan \beta$. In the usual decoupling region with large $m_{A}$, the light CP-even Higgs $h^{0}$ is SMlike while the other Higgses are almost degenerate: $m_{H^{0}} \sim m_{A} \sim m_{H^{ \pm}}$. Thus, $A \rightarrow Z H^{0}$ or $H^{0} \rightarrow Z A$ is not allowed kinematically. $A \rightarrow Z h^{0}$ is typically suppressed by the small coupling: $\cos (\beta-\alpha) \sim 0$, and is only relevant for small $\tan \beta$. In the NMSSM, the Higgs sector of MSSM is enlarged to include an additional singlet. It was shown in ref. [17] that there are regions of parameter space where the decay $A_{i} \rightarrow H_{j} Z$ can be significant.

\section{Current experimental limits}

Searches for the non-SM like Higgses, mainly in the $b b, \mu \mu, \tau \tau$ or $W W / Z Z$ channels have been performed both by ATLAS and CMS. No evidence for a neutral non-SM like Higgs was found.

Searches for the neutral Higgs bosons $\Phi$ of the MSSM in the process $p p \rightarrow \Phi \rightarrow$ $\mu^{+} \mu^{-} / \tau^{+} \tau^{-}$have been performed by the ATLAS [24], and in the $\tau^{+} \tau^{-}$channel at CMS [25]. Limits in the $\mu \mu$ channel are much weaker given the extremely small branching fraction in the MSSM. The production mechanisms considered were both gluon fusion and $b b$ associated production, and the exclusion results were reported for the MSSM $m_{h}^{\max }$ scenario. The ATLAS study was performed at $\sqrt{s}=7 \mathrm{TeV}$ with $4.7-4.8 \mathrm{fb}^{-1}$ integrated luminosity looking at three different possible $\tau \tau$ final states, $\tau_{e} \tau_{\mu}, \tau_{\text {lep }} \tau_{\text {had }}$, and $\tau_{\text {had }} \tau_{\text {had }}$. The ATLAS search rules out a fairly sizable portion of the MSSM parameter space, extending from about $\tan \beta$ of 10 for $m_{A} \sim 130 \mathrm{GeV}$, to $\tan \beta \approx 60$ for $m_{A}=500 \mathrm{GeV}$. The corresponding exclusion in $\sigma_{\Phi} \times \mathrm{BR}(\Phi \rightarrow \tau \tau)$ extends from roughly $40 \mathrm{pb}$ to $0.3 \mathrm{pb}$ in that mass range. The CMS study was performed with $19.7 \mathrm{fb}^{-1}$ integrated luminosity at $8 \mathrm{TeV}$ and $4.9 \mathrm{fb}^{-1}$ at $7 \mathrm{TeV}$ in the $\tau_{e} \tau_{\mu}, \tau_{\mu} \tau_{\mu}, \tau_{\text {lep }} \tau_{\text {had }}$, and $\tau_{\text {had }} \tau_{\text {had }}$ final states. The search excludes roughly between $\tan \beta$ of 4 for $m_{A}=140 \mathrm{GeV}$ and $\tan \beta \approx 60$ for $m_{A}=1000 \mathrm{GeV}$. The corresponding exclusion in $\sigma_{\Phi} \times \mathrm{BR}(\Phi \rightarrow \tau \tau)$ extends from roughly $2 \mathrm{pb}$ to $13 \mathrm{fb}$ in that mass range.

In figure 1, we recast the current 95\% C.L. limit of $p p \rightarrow \Phi \rightarrow \tau^{+} \tau^{-}$in the $\left(m_{A}, \tan \beta\right)$ parameter space of the Type II 2HDM [25] (left panel) and the projected $5 \sigma$ reach at the $14 \mathrm{TeV}$ LHC with $30 \mathrm{fb}^{-1}$ luminosity [26] (right panel). In both plots, the solid black curves correspond to the limits in the MSSM, when $m_{A} \approx m_{H^{0}}$ with both $A$ and $H^{0}$ contributing to the signal. The solid red curves correspond to the limits in the type II 2HDM, when only contribution from $A$ is included and $H^{0}$ is decoupled. The reach is considerably weaker: the current exclusion is about $\tan \beta \sim 12$ at $m_{A}=160 \mathrm{GeV}$, and $\tan \beta \sim 46$ for $m_{A}=600 \mathrm{GeV}$. At the $14 \mathrm{TeV}$ LHC with $30 \mathrm{fb}^{-1}$ luminosity, the $5 \sigma$ reach extends beyond the current exclusion for large $m_{A}$. Dashed lines indicate the reduced reach in the $\tau \tau$ channel once $A \rightarrow h^{0} Z$ mode opens, for a benchmark point of $\sin (\beta-\alpha)=0$, $m_{h^{0}}=50 \mathrm{GeV}$ and $m_{H^{0}}=126 \mathrm{GeV}$.

Searches with $b b$ final states have also been performed for the MSSM Higgs in the associated production $p p \rightarrow b \Phi+X$. The CMS search, done with $2.7-4.8 \mathrm{fb}^{-1}$ of data at 

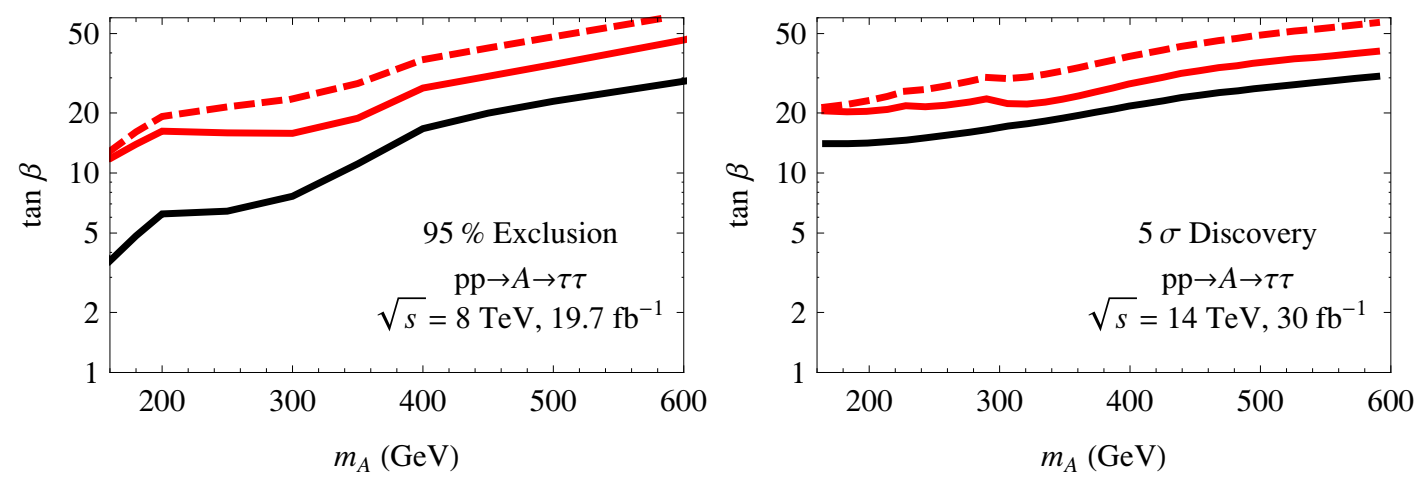

Figure 1. The reach of $p p \rightarrow A \rightarrow \tau \tau$ in $m_{A}-\tan \beta$ parameter space of the Type II 2HDM. Left panel shows the current $95 \%$ C.L. exclusion limits from CMS [25] with $19.7 \mathrm{fb}^{-1}$ data collected at the $\sqrt{s}=8 \mathrm{TeV}$ LHC. Right panel shows the projected $5 \sigma$ discovery reach at the $14 \mathrm{TeV}$ LHC with $30 \mathrm{fb}^{-1}$ luminosity [26]. In both plots, the solid black curves correspond to the limits in the MSSM, when $m_{A} \approx m_{H^{0}}$ with both $A$ and $H^{0}$ contributing to the signal. The solid red curves correspond to the limits in the type II $2 \mathrm{HDM}$, when only contribution from $A$ is included and $H^{0}$ is decoupled. Also shown in the red dashed curves are the reduced $\tau \tau$ channel limits when $A \rightarrow h^{0} Z$ is open with the parameter choice of $\sin (\beta-\alpha)=0, m_{h^{0}}=50 \mathrm{GeV}$ and $m_{H^{0}}=126 \mathrm{GeV}$.

$\sqrt{s}=7 \mathrm{TeV}$ excludes $\tan \beta$ values between 18 and 42 in the mass range $90 \mathrm{GeV}<m_{A}<$ $350 \mathrm{GeV}[27]$.

The ATLAS collaboration has also looked for the heavier CP-even Higgs in the Type I and Type II 2HDM, assuming the lighter CP-even Higgs is the discovered $126 \mathrm{GeV}$ boson [28]. The study was performed with $13 \mathrm{fb}^{-1}$ integrated luminosity at $8 \mathrm{TeV}$ and considered both gluon fusion and vector boson fusion production. Searches in the process $H^{0} \rightarrow W W \rightarrow e \mu \nu_{e} \nu_{\mu}$ exclude a significant region of the $m_{H^{0}}-\cos \alpha$ parameter space in the mass range $135 \mathrm{GeV}<m_{H^{0}}<200 \mathrm{GeV}$ for the Type II 2HDM. The excluded region shrinks for higher $\tan \beta$ due to the reduced branching ratio to $W W$. This would serve as a useful constraint if we were to look at decays of the relatively light $H^{0}$ to light $A$ 's. In this paper, we consider values of $m_{H}$ outside this mass range so this constraint does not apply.

The CMS collaboration has also searched for the heavier CP-even Higgs $H^{0}$ and a heavy CP-odd Higgs $A$ in $2 \mathrm{HDM}$ via the processes $g g \rightarrow A \rightarrow h^{0} Z$ and $g g \rightarrow H^{0} \rightarrow$ $h^{0} h^{0}$, assuming the lighter Higgs $h^{0}$ is the discovered $126 \mathrm{GeV}$ boson [29]. The study was performed with $19.5 \mathrm{fb}^{-1}$ integrated luminosity at $8 \mathrm{TeV}$. Various possible decays of the SM-Higgs were taken into account. Assuming SM branching ratios for $h^{0}$, this study gives an upper bound on $\sigma \times \operatorname{BR}\left(A \rightarrow h^{0} Z\right)$ of roughly $1.5 \mathrm{pb}$ for $m_{A}$ between 260 and $360 \mathrm{GeV}$ and $\sigma \times \mathrm{BR}\left(H^{0} \rightarrow h^{0} h^{0}\right)$ between $8 \mathrm{pb}$ and $6 \mathrm{pb}$ for masses $m_{H^{0}}$ between $260 \mathrm{GeV}$ and $360 \mathrm{GeV}$. The corresponding excluded parameter space for the Type II 2HDM in the $\tan \beta-\cos (\beta-\alpha)$ plane was also analyzed. In the analysis presented in this paper, we do not necessarily require that the daughter Higgs in $A \rightarrow H Z$ to be the SM-like Higgs or have SM-like branching ratios. Furthermore we also analyze the process $H \rightarrow A Z$ for light $A$ and its implication in the Type II 2HDM. 


\section{Collider analysis}

In this section, we will present model independent limits on the $\sigma \times \mathrm{BR}$ for both 95\% C.L. exclusion and $5 \sigma$ discovery for $A / H \rightarrow H Z / A Z$ in the various final states of $b b \ell \ell, \tau \tau \ell \ell$ and $Z Z Z(4 \ell 2 j)$. In this study we focus on the leptonic decay of the $Z$, which allows precise mass reconstruction and suppresses the background sufficiently. Other decay modes of the $Z$, for example $Z \rightarrow \tau \tau$, might be useful in studying this channel as well. In the discussion of the analyses and results below, we use the decay $A \rightarrow H Z$ for $m_{A}>m_{H}+m_{Z}$ as an illustration. Since we do not make use of angular correlations, the bounds obtained for $A \rightarrow H Z$ apply to $H \rightarrow A Z$ as well with the values of $m_{A}$ and $m_{H}$ switched.

\section{1 $\quad A / H \rightarrow H Z / A Z \rightarrow b b \ell \ell$}

We start our analysis by looking at the channel $A / H \rightarrow H Z / A Z \rightarrow$ bbll for $\ell=e, \mu$, focusing only on the gluon fusion production channels. We use $H$ to refer to either the light or the heavy CP-even Higgs. Since the only allowed couplings are of the type $H-A-Z$, if the parent particle is a scalar $H$, the daughter particle is necessarily a pseudoscalar $A$ and vice versa.

The dominant SM backgrounds for bbll final states are $Z / \gamma^{*} b b$ with leptonic $Z / \gamma^{*}$ decay, $t \bar{t}$ with leptonically decaying top quarks, $Z Z \rightarrow b b \ell \ell$, and $H_{\mathrm{SM}} Z$ [30-33]. We have ignored the subdominant backgrounds from $W Z, W W, H_{\mathrm{SM}} \rightarrow Z Z, W b b$, Multijet QCD Background, $Z j j, Z \ell \ell$ as well as $t W b$. These backgrounds either have small production cross sections, or can be sufficiently suppressed by the cuts imposed. We have included $H_{\mathrm{SM}} Z$ here even if the cross section is very small because it has the same final state as the process under consideration, especially for the $A \rightarrow H_{\mathrm{SM}} Z$ case. The total cross sections for these backgrounds can be found in table 1 .

We use Madgraph 5/MadEvent v1.5.11 [34] to generate our signal and background events. These events are passed to Pythia v2.1.21 [35] to simulate initial and final state radiation, showering and hadronization. The events are further passed through Delphes 3.09 [36] with the Snowmass combined LHC detector card [37] to simulate detector effects.

For the signal process, we generated event samples at the $14 \mathrm{TeV}$ LHC for $g g \rightarrow A \rightarrow$ $H Z$ with the daughter particle mass fixed at 50,126 , and $200 \mathrm{GeV}$ while varying the parent particle mass in the range of $150-600 \mathrm{GeV}$. We applied the following cuts to identify the signal from the backgrounds: ${ }^{3}$

1. Two isolated leptons, two tagged b's.

$$
n_{\ell}=2, n_{b}=2 \text {, with }\left|\eta_{\ell, b}\right|<2.5, p_{T, \ell}>10 \mathrm{GeV}, p_{T, b}>15 \mathrm{GeV} .
$$

For jet reconstruction, the anti- $k_{T}$ jet algorithm with $R=0.5$ is used.

2. Lepton trigger [38, 39].

$$
p_{T, \ell_{1}}>30 \mathrm{GeV} \text { or } p_{T, \ell_{1}}>20 \mathrm{GeV}, p_{T, \ell_{2}}>10 \mathrm{GeV} .
$$

\footnotetext{
${ }^{3}$ Requiring the missing transverse energy to be small would potentially greatly reduce the $t \bar{t}$ background. However, including pile-up effects introduces $E_{T}$ in the signal events, which renders the cut inefficient. We thank Meenakshi Narain and John Stupak for pointing this out to us.
} 

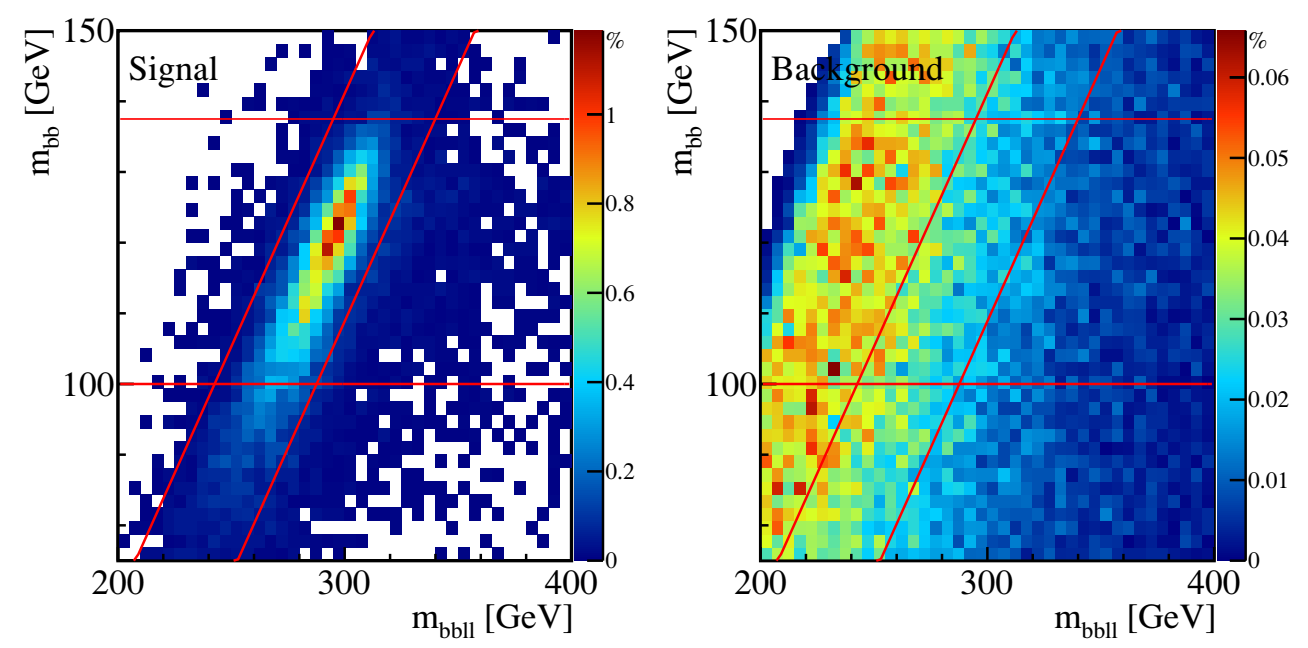

Figure 2. Normalized distribution (in percent as given by the color code along the $y$-axis) of $m_{b b}$ versus $m_{b b \ell \ell}$ for the signal (left panel), and the backgrounds $\left(Z / \gamma^{*} b b+Z Z+H_{\mathrm{SM}}+t \bar{t}\right)$ (right panel) for $m_{A}=300 \mathrm{GeV}$ and $m_{H}=126 \mathrm{GeV}$. Two horizontal lines indicate the $m_{b b}$ range and two slanted lines indicate the $m_{b b \ell \ell}$ range, as given in eq. (4.4).

3. Dilepton mass $m_{\ell \ell}$. We require the dilepton mass to be in the $Z$-mass window:

$$
80 \mathrm{GeV}<m_{\ell \ell}<100 \mathrm{GeV} .
$$

4. $m_{b b}$ versus $m_{b b \ell \ell}$. We require the dijet mass $m_{b b}$ to be close to the daughter-Higgs mass $m_{H}$ and the mass $m_{b b \ell \ell}$ to be close to the parent-Higgs mass $m_{A}$. These two invariant masses are correlated, i.e., if we underestimate $m_{b b}$ we also underestimate $m_{b b \ell \ell}$. To take this into account we apply a two-dimensional cut:

$$
\begin{aligned}
\left(0.95-w_{b b}\right) \times m_{H}<m_{b b} & <\left(0.95+w_{b b}\right) \times m_{H} \text { with } w_{b b}=0.15, \\
\frac{m_{Z}+m_{H}}{m_{A}} \times\left(m_{b b \ell \ell}-m_{A}-w_{b b \ell \ell}\right) & <m_{b b}-m_{H}<\frac{m_{Z}+m_{H}}{m_{A}} \times\left(m_{b b \ell \ell}-m_{A}+w_{b b \ell \ell}\right),
\end{aligned}
$$

where $w_{b b} \times m_{H}$ is the width of the dijet mass window. Note that the slightly shifted reconstructed Higgs mass $m_{b b}\left(0.95 m_{H}\right.$ instead of $\left.m_{H}\right)$ is due to the reconstruction of the $b$-jet with a small size of $R=0.5$. The second condition describes two lines going through the points $\left(m_{A} \pm w_{b b \ell \ell}, m_{H}\right)$ with slope $\left(m_{Z}+m_{H}\right) / m_{A}$. We choose a width for the $m_{b b \ell \ell}$ peak of $w_{b b \ell \ell}=\operatorname{Max}\left(\Gamma_{H_{\mathrm{SM}}} \mid m_{A}, 0.075 m_{A}\right)$ where $\left.\Gamma_{H_{\mathrm{SM}}}\right|_{m_{A}}$ is the width of a SM Higgs with mass $m_{A}$ [40]. This accounts for both small Higgs masses for which the width of the peak is caused by detector effects and large Higgs masses for which the physical width dominates.

The effectiveness of this cut is shown in figure 2 for $m_{A}=300 \mathrm{GeV}$ and $m_{H}=$ $126 \mathrm{GeV}$, with two horizontal lines indicating the $m_{b b}$ range and two slanted lines indicating the $m_{b b \ell \ell}$ range as given in eq. (4.4). Left and right panels show the normalized distributions for the signal and the backgrounds, respectively. The color coding is such that points in dark red are most likely, with the probability falling as 

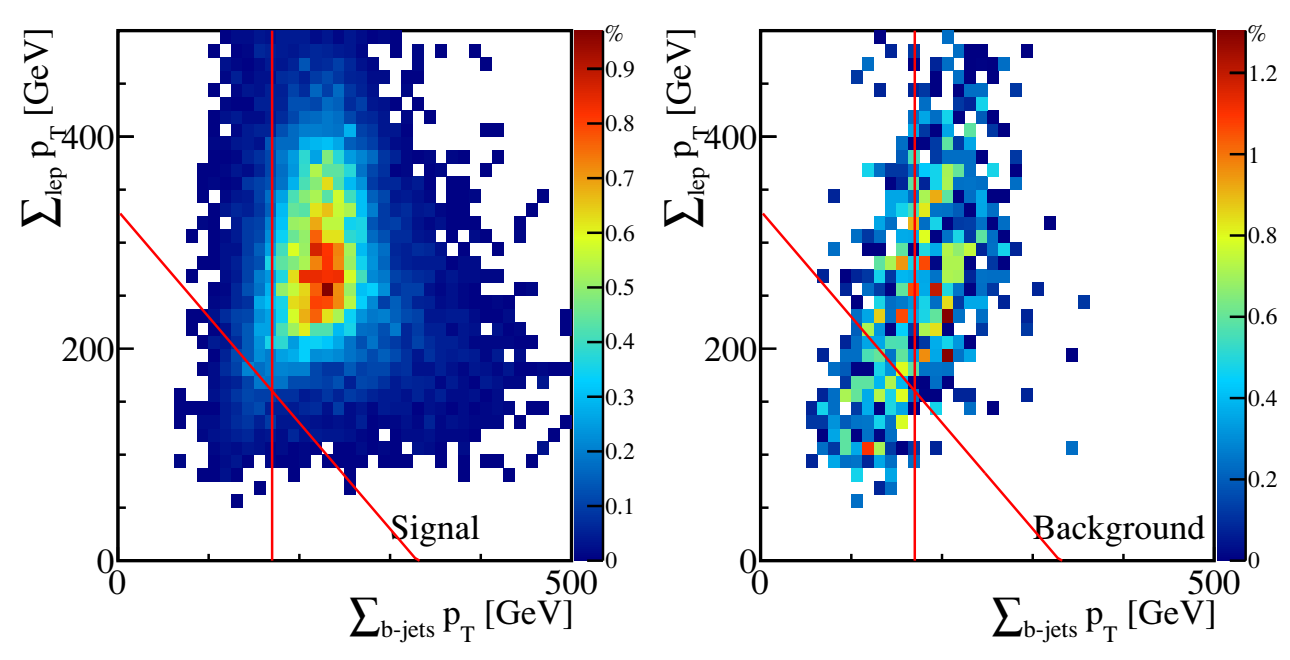

Figure 3. Normalized transverse momentum distribution $\sum_{\ell} p_{T}$ versus $\sum_{b \text { jets }} p_{T}$ for the signal (left panel) and the backgrounds (right panel) for $m_{A}=500 \mathrm{GeV}$ and $m_{H}=126 \mathrm{GeV}$. Two red lines indicate the conditions used in the cuts as given in eq. (4.5).

we reach dark blue as indicated on the right color panel in each plot. The numbers in this panel represent the percentage of the number of events that survive in each bin for the corresponding color. The signal region in each plot is the region bounded by the two pairs of slanting and horizontal lines. As expected, we see that most of the signal events fall within this strip, while the backgrounds mostly lie outside it.

5. Transverse momentum. We require the sum of the transverse momenta of the bottom jets and the sum of the transverse momenta of the bottom jets and leptons to satisfy:

$$
\begin{aligned}
\sum_{b \text { jets }} p_{T} & >0.6 \times \frac{m_{A}^{2}+m_{H}^{2}-m_{Z}^{2}}{2 m_{A}}, \\
\sum_{\ell, b \text { jets }} p_{T} & >0.66 \times m_{A} .
\end{aligned}
$$

The cuts given in eq. (4.5) follow from simple relativistic kinematics applied to the process as applicable to the entire momenta, i.e., $\sum_{b \text { jets }} p_{b_{i}}=\frac{m_{A}^{2}+m_{H}^{2}-m_{Z}^{2}}{2 m_{A}}$ assuming that the parent Higgs $A$ is at rest. We have chosen to specialize this formula to the transverse part alone, including an optimization factor of 0.6. In figure 3 , we show how this $p_{T}$ cut helps in extracting the signal over the backgrounds for the case where the parent mass is $500 \mathrm{GeV}$ and the daughter mass is $126 \mathrm{GeV}$. The regions of the plot to the left of the two lines are excluded. It can be seen that while the signal is largely intact, a good portion of the backgrounds gets cut out.

In table 1, we show the signal and background cross sections with cuts for signal benchmark point of $m_{A}=300 \mathrm{GeV}$ and $m_{H}=126 \mathrm{GeV}$ at the $14 \mathrm{TeV}$ LHC. We have chosen a nominal value for $\sigma \times \mathrm{BR}(g g \rightarrow A / H \rightarrow H Z / A Z \rightarrow b b \ell \ell)$ of $100 \mathrm{fb}$ to illustrate the cut efficiencies for the signal process. In the last column, $S / \sqrt{B}$ is shown for an integrated 


\begin{tabular}{|l|c|ccccc|}
\hline Cut & Signal [fb] & $b b \ell \ell[\mathrm{fb}]$ & $H_{\mathrm{SM}} Z[\mathrm{fb}]$ & $t \bar{t}[\mathrm{fb}]$ & $S / B$ & $S / \sqrt{B}$ \\
\hline$\sigma_{\text {total }}$ & & $2.21 \times 10^{6}$ & 883 & $9.20 \times 10^{5}$ & - & - \\
Leptonic decay & 100 & $2.21 \times 10^{6}$ & 59.4 & $2.15 \times 10^{4}$ & - & - \\
Two leptons, two b's [eq. (4.1)] & 6.35 & 343 & 3.44 & 1409 & 0.0036 & 2.63 \\
Lepton trigger [eq. (4.2)] & 6.35 & 336 & 3.44 & 1394 & 0.0037 & 2.65 \\
$m_{\ell \ell}$ [eq. (4.3)] & 5.76 & 285 & 3.13 & 189 & 0.012 & 4.59 \\
$m_{b b}$ vs $m_{b b \ell \ell}[$ eq. (4.4)] & 3.03 & 11.5 & 0.401 & 11.5 & 0.14 & 11.5 \\
$\sum p_{T, b}, \sum\left(p_{T, b}+p_{T, \ell}\right)[$ eq. (4.5)] & 2.81 & 8.11 & 0.361 & 8.38 & 0.17 & 12.0 \\
\hline
\end{tabular}

Table 1. Signal and background cross sections with cuts for the signal benchmark point $m_{A}=$ $300 \mathrm{GeV}$ and $m_{H}=126 \mathrm{GeV}$ at the $14 \mathrm{TeV}$ LHC. We have chosen a nominal value for $\sigma \times \operatorname{BR}(g g \rightarrow$ $A \rightarrow H Z \rightarrow b b \ell \ell$ ) of $100 \mathrm{fb}$ to illustrate the cut efficiencies for the signal process. In the last column, $S / \sqrt{B}$ is shown for an integrated luminosity of $\mathcal{L}=300 \mathrm{fb}^{-1}$.
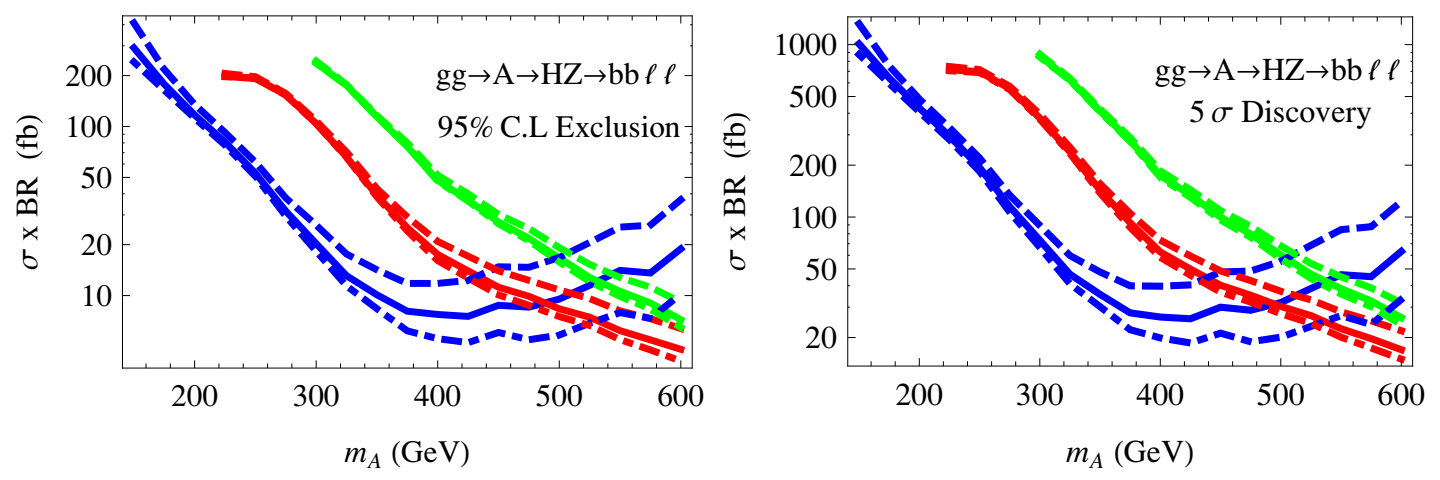

Figure 4. The $95 \%$ C.L. exclusion (left) and $5 \sigma$ discovery (right) limits for $\sigma \times \operatorname{BR}(g g \rightarrow A \rightarrow$

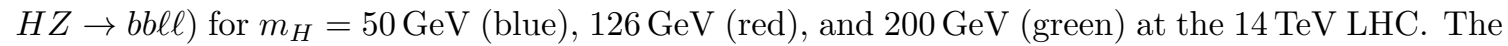
dashed, solid and dot-dashed lines correspond to an integrated luminosity of 100, 300 and $1000 \mathrm{fb}^{-1}$, respectively. Here, we have assumed a $10 \%$ systematic error on the backgrounds. These results are equally applicable to the $H \rightarrow A Z$ process for the same parent and daughter Higgs masses.

luminosity of $\mathcal{L}=300 \mathrm{fb}^{-1}$. Note that for both the signal and the backgrounds, the biggest reduction of the cross sections arises upon demanding exactly two isolated leptons and $b$ jets. In fact, the signal cross section drops from $100 \mathrm{fb}$ to $6.35 \mathrm{fb}$ at this stage. The two $b$ tag efficiencies bring down the cross section by $0.7^{2} \approx 50 \%$. Other contributing factors are leptons and $b$ jets that are either soft or in the forward direction, or non-isolated leptons and $b$ jets. We also remark that the $m_{\ell \ell}$ cut does not have a significant effect on either the signal or the $b b \ell \ell$ and $H_{\mathrm{SM}} Z$ backgrounds since these are dominated by the leptons coming from $Z$, but does have a pronounced effect on the $t \bar{t}$ background. The second to last row clearly demonstrates the efficacy of the two dimensional cut in the $m_{b b}-m_{b b \ell \ell}$ plane.

In figure 4, we display the results at the $14 \mathrm{TeV}$ LHC for 95\% C.L. exclusion (left panel) and $5 \sigma$ discovery (right panel) limits for $\sigma \times \mathrm{BR}(g g \rightarrow A \rightarrow H Z \rightarrow b b \ell \ell)$, which applies for $H \rightarrow A Z$ as well with $m_{A}$ and $m_{H}$ switched. The blue, red, and green curves correspond to the daughter particle being $50 \mathrm{GeV}, 126 \mathrm{GeV}$, and $200 \mathrm{GeV}$, respectively. The masses of the daughter particle are chosen such that they represent cases with a light Higgs, a SM-like Higgs, as well as a heavy Higgs that can decay to $W W / Z Z$. For each mass, 
we have displayed the results for three luminosities: $100 \mathrm{fb}^{-1}$ (dashed), $300 \mathrm{fb}^{-1}$ (solid), and $1000 \mathrm{fb}^{-1}$ (dot-dashed), with $10 \%$ systematic error included [41]. Better sensitivity is achieved for larger $m_{A}$ since the mass cuts on $m_{b b}$ and $m_{b b \ell \ell}$ have a more pronounced effect on SM backgrounds for larger masses. The limit, however, gets worse for the $m_{H}=$ $50 \mathrm{GeV}$ case when $m_{A} \gtrsim 400 \mathrm{GeV}$ (blue curves). This is due to the decrease of the signal cut efficiency for a highly boosted daughter particle with two collimated $b$ jets. For the interesting case where the daughter particle is $126 \mathrm{GeV}$, it is seen that the discovery limits for a $300 \mathrm{fb}^{-1}$ collider fall from about $0.7 \mathrm{pb}$ for $m_{A}$ of $225 \mathrm{GeV}$, to less than $20 \mathrm{fb}$ for a $600 \mathrm{GeV}$ parent particle. These numbers do not change appreciably between the three chosen luminosity values, except for the case of $m_{H}=50 \mathrm{GeV}$ and $m_{A} \gtrsim 400 \mathrm{GeV}$. This is because we have chosen a uniform $10 \%$ systematic error on the backgrounds, which dominates the statistical errors for most of the parameter region. For a given parent particle mass $m_{A}$, limits are better for smaller $m_{H}=50 \mathrm{GeV}$. This is because the $m_{b b}$ distribution for the dominating $Z b b$ and $t t$ backgrounds peaks around higher masses $m_{b b} \approx$ $70-200 \mathrm{GeV}$ and therefore the background rejection efficiency for $m_{b b} \approx 50 \mathrm{GeV}$ is high. For $m_{H}=126$ and $200 \mathrm{GeV}$ the background rejection efficiencies are comparable but for $m_{H}=200 \mathrm{GeV}$ the signal cut efficiency is worse and hence the exclusion limits are the highest for $m_{H}=200 \mathrm{GeV}$.

We reiterate here these exclusion and discovery limits are completely model independent. Whether or not discovery/exclusion is actually feasible in this channel should be answered within the context of a particular model, in which the theoretically predicted cross sections and branching fractions can be compared with the exclusion or discovery limits. We will do this in section 5 using Type II $2 \mathrm{HDM}$ as a specific example.

\section{2 $\quad A / H \rightarrow H Z / A Z \rightarrow \tau \tau \ell \ell$}

We now turn to the process $g g \rightarrow A / H \rightarrow H Z / A Z \rightarrow \tau \tau \ell \ell$. Since we want to reconstruct the final state particles unambiguously, we will employ $\tau$ tags and thus will only consider fully hadronic $\tau$ decays. While the signal is typically suppressed compared to the bbll case due to the smaller $H \rightarrow \tau \tau$ branching fraction, the SM backgrounds [32, 33] are much smaller due to the absence of $b$ jets in the final states. The dominant background is $Z Z$. We have also included $H_{\mathrm{SM}} Z$ background even though it is negligible for most cases.

Here, we list the cuts employed:

1. Two isolated leptons and two tagged $\tau$ 's.

$$
n_{\ell}=2, n_{\tau}=2 \text {, with }\left|\eta_{\ell, \tau}\right|<2.5, p_{T, \ell}>10 \mathrm{GeV}, p_{T, \tau}>20 \mathrm{GeV} .
$$

We do not impose jet veto.

2. Lepton trigger.

$$
p_{T, \ell_{1}}>30 \mathrm{GeV} \text { or } p_{T, \ell_{1}}>20 \mathrm{GeV}, p_{T, \ell_{2}}>10 \mathrm{GeV} .
$$

3. Dilepton mass $m_{\ell \ell}$.

$$
80 \mathrm{GeV}<m_{\ell \ell}<100 \mathrm{GeV} .
$$



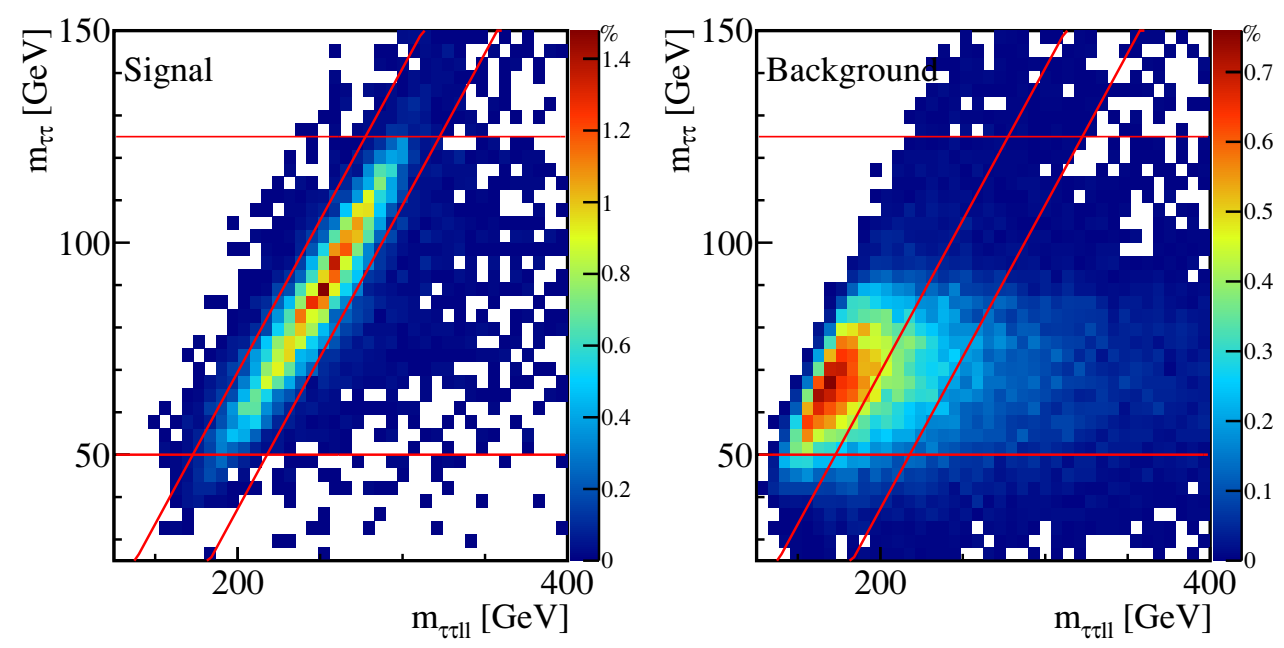

Figure 5. Normalized distribution of $m_{\tau \tau}$ versus $m_{\tau \tau \ell \ell}$ for the signal (left panel), the backgrounds (right panel) for $m_{A}=300 \mathrm{GeV}$ and $m_{H}=126 \mathrm{GeV}$. Two horizontal lines indicate the $m_{\tau \tau}$ range and two slanted lines indicate the $m_{\tau \tau \ell \ell}$ range, as given in eq. (4.9).

4. $m_{\tau \tau}$ versus $m_{\tau \tau \ell \ell}$. The expected Higgs mass is shifted more towards smaller values compared to the $b b$ case. This is because of the hadronic decay of $\tau$ with missing energy carried away by neutrinos. Our 2-D cuts are modified as follows:

$$
\begin{aligned}
\left(0.7-w_{\tau \tau}\right) \times m_{H}<m_{\tau \tau} & <\left(0.7+w_{\tau \tau}\right) \times m_{H} \text { with } w_{\tau \tau}=0.3 ; \\
\frac{m_{Z}+m_{H}}{m_{A}} \times\left(m_{\tau \tau \ell \ell}-m_{A}-w_{\tau \tau \ell \ell}\right) & <m_{\tau \tau}-m_{H}<\frac{m_{Z}+m_{H}}{m_{A}} \times\left(m_{\tau \tau \ell \ell}-m_{A}+w_{\tau \tau \ell \ell}\right),
\end{aligned}
$$

with $w_{\tau \tau \ell \ell}=\operatorname{Max}\left(\left.\Gamma_{H_{\mathrm{SM}}}\right|_{m_{A}}, 0.075 m_{A}\right)$. We show the normalized 2-D distribution as well as cuts imposed as indicated by red lines in figure 5 for the signal (left panel) and the backgrounds (right panel). The cut filters out most of the backgrounds while retaining the signal, yielding a good $S / \sqrt{B}$ value.

5. Transverse momentum.

$$
\begin{aligned}
\sum_{\tau} p_{T} & >0.4 \times \frac{m_{A}^{2}+m_{H}^{2}-m_{Z}^{2}}{2 m_{A}}, \\
\sum_{\ell, \tau} p_{T} & >0.66 \times m_{A} .
\end{aligned}
$$

The looser cut on $\sum_{\tau} p_{T}$ compared to the bbll case is again due to the extra missing $E_{T}$ in the $\tau$ decay.

In table 2, we present the cross sections after the individual cut is imposed sequentially. We take a nominal signal cross section of $10 \mathrm{fb}$ to illustrate the efficiency of the chosen cuts. Again, the 2-D $m_{\tau \tau}-m_{\tau \tau \ell \ell}$ cut improves the $S / \sqrt{B}$ value significantly.

In figure 6 , we show the $95 \%$ C.L. exclusion and $5 \sigma$ discovery reach in $\sigma \times \mathrm{BR}(g g \rightarrow$ $A \rightarrow H Z \rightarrow \tau \tau \ell \ell)$ for the $14 \mathrm{TeV}$ LHC. The general feature of these plots follows that of 


\begin{tabular}{|l|c|cccc|}
\hline Cut & Signal [fb] & $\tau \tau \ell \ell[\mathrm{fb}]$ & $H_{\mathrm{SM}} Z[\mathrm{fb}]$ & $S / B$ & $S / \sqrt{B}$ \\
\hline$\sigma_{\text {total }}$ & & 218 & 883 & - & - \\
leptonic decay & 10 & 218 & 3.02 & - & - \\
Two leptons, two $\tau$ 's [eq. (4.6)] & 0.43 & 1.622 & 0.1136 & 0.2684 & 5.921 \\
Lepton trigger [eq. (4.7)] & 0.43 & 1.572 & 0.1134 & 0.2768 & 6.011 \\
$m_{\ell \ell}$ [eq. (4.8)] & 0.39 & 1.312 & 0.1031 & 0.301 & 5.869 \\
$m_{\tau \tau}$ vs $m_{\tau \tau \ell \ell}[$ eq. (4.9)] & 0.29 & 0.3029 & 0.023 & 0.9643 & 9.192 \\
$\sum p_{T, \tau}, \sum\left(p_{T, \tau}+p_{T, \ell}\right)[$ eq. (4.10)] & 0.18 & 0.064 & 0.013 & 2.872 & 12.68 \\
\hline
\end{tabular}

Table 2. Signal and background cross sections with cuts for signal benchmark point of $m_{A}=$ $300 \mathrm{GeV}$ and $m_{H}=126 \mathrm{GeV}$ at the $14 \mathrm{TeV}$ LHC. We have chosen a nominal value for $\sigma \times \operatorname{BR}(g g \rightarrow$ $A \rightarrow H Z \rightarrow \tau \tau \ell \ell)$ of $10 \mathrm{fb}$ to illustrate the cut efficiencies for the signal process. In the last column, $S / \sqrt{B}$ is shown for an integrated luminosity of $\mathcal{L}=300 \mathrm{fb}^{-1}$.
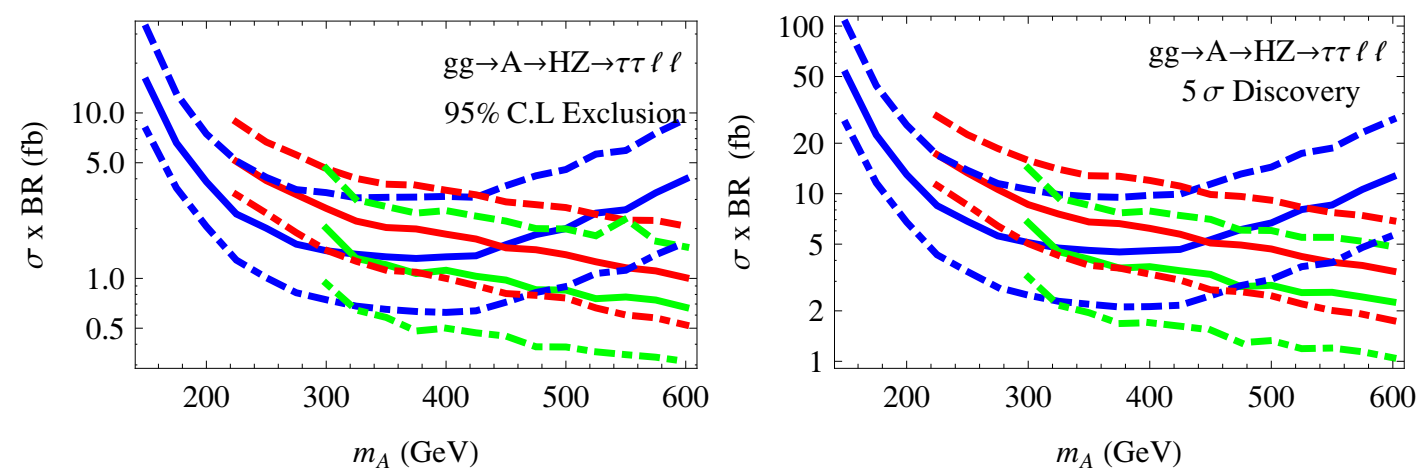

Figure 6. The 95\% C.L. exclusion (left) and $5 \sigma$ discovery (right) limits for $\sigma \times \operatorname{BR}(g g \rightarrow A \rightarrow$ $H Z \rightarrow \tau \tau \ell \ell$ ) for $m_{H}=50 \mathrm{GeV}$ (blue), $126 \mathrm{GeV}$ (red), and $200 \mathrm{GeV}$ (green) at the $14 \mathrm{TeV} \mathrm{LHC}$. The dashed, solid and dot-dashed lines correspond to an integrated luminosity of 100, 300 and $1000 \mathrm{fb}^{-1}$, respectively. A $10 \%$ systematic error on the backgrounds is assumed as well.

figure 4, particularly with highly boosted daughter particles making $\tau$ identification more challenging, as shown by the blue curves for $50 \mathrm{GeV}$ daughter particle mass, which exhibit worse limits for $m_{A}>400 \mathrm{GeV}$. The exclusion limits are lowest for small $m_{H}=50 \mathrm{GeV}$ and also for high $m_{H}=200 \mathrm{GeV}$ since the dominating $Z Z$ background peaks at $m_{\tau \tau} \approx 90 \mathrm{GeV}$ and therefore our $m_{\tau \tau}$ mass cut leads to a high background rejection for lower or higher $m_{H}$. Since the statistical error dominates the $10 \%$ systematic error, the $\sigma \times \mathrm{BR}$ limits scale roughly with $1 / \sqrt{L}$, as indicated by the dashed, solid and dot-dashed lines for different luminosities.

Compared to the bbll case, the $\sigma \times \mathrm{BR}$ reach in $\tau \tau \ell \ell$ case is better due to significantly lower SM backgrounds. For the $126 \mathrm{GeV}$ daughter particle case with $300 \mathrm{fb}^{-1}$, the $5 \sigma$ discovery reach varies from about $20 \mathrm{fb}$ for parent mass of $225 \mathrm{GeV}$ to about $3 \mathrm{fb}$ for $600 \mathrm{GeV}$. Thus, given the typical ratio of $\operatorname{Br}(H / A \rightarrow b b): \operatorname{Br}(H / A \rightarrow \tau \tau) \sim 3 m_{b}^{2} / m_{\tau}^{2}$, the reach in $\tau \tau \ell \ell$ can be comparable or even better than $b b \ell \ell$ channel, in particular, for smaller parent Higgs masses. 


\section{3 $\quad A \rightarrow H Z \rightarrow Z Z Z \rightarrow 4 \ell+2 j$}

We now consider the case where the daughter particle decays to a pair of $Z$ bosons, which only applies to $A \rightarrow H Z \rightarrow Z Z Z$. This process involves a trade-off between having a clean final state with suppressed backgrounds and suppressed signal cross section for detection. We find that the best final states combination that yields signal cross sections that are not too suppressed in realistic models with controllable backgrounds is the $4 \ell+2 j$ final state: $A \rightarrow H Z \rightarrow Z Z Z \rightarrow 4 \ell+2 j$. The SM backgrounds for this process come from the single, double and triple vector boson processes including additional jets as well as $t \bar{t}$ background [37, 42, 43].

Note that the $Z$ 's from the $H$ decay could be either on-shell or off-shell depending on $m_{H}$. We will display our results for two cases: one where one of the final state $Z$ 's is necessarily off-shell, and another where both are on-shell. We will find that the latter case leads to much better discovery prospects.

We applied the following set of cuts:

- Four isolated leptons, two jets.

$$
n_{\ell}=4, n_{j} \geq 2 \text {, with }\left|\eta_{\ell}\right|<2.5, p_{T, \ell}>10 \mathrm{GeV},\left|\eta_{j}\right|<5, p_{T, j}>20 \mathrm{GeV} \text {. }
$$

For jet reconstruction, we use the anti- $k_{T}$ jet algorithm with $R=0.5$. We also require the leptons to satisfy the lepton trigger as in eq. (4.2).

- Three $Z$-candidates. We reconstruct the hadronically decaying $Z$ using the 2 hardest jets. To reconstruct the leptonically decaying $Z$ 's:

- 4e or $4 \mu$. If we have $4 e$ or $4 \mu$, we first find the combination of electrons or muons with opposite charge that is closest to the $Z$-mass. The other 2 electrons or muons are combined to find the last $Z$.

$-2 e 2 \mu$. Here, we combine the same flavored leptons in a straightforward manner.

- $Z$ masses. We require the hardonically decaying $Z_{1}$, the well reconstructed leptonically decaying $Z_{2}$ and the final reconstructed leptonically decaying $Z_{3}$ to be in the following windows:

$$
\begin{aligned}
60 \mathrm{GeV} & <m_{Z_{1}}<115 \mathrm{GeV} . \\
80 \mathrm{GeV} & <m_{Z_{2}}<100 \mathrm{GeV} . \\
m_{\text {min }} & <m_{Z_{3}}<115 \mathrm{GeV} .
\end{aligned}
$$

Here, we assume $Z_{1}$ to be on-shell. However, we allow for the possibility that $Z_{3}$ could be far off-shell. The $m_{\text {min }}$ employed here mimics the LHC search strategy for the SM Higgs, and its value depends on the Higgs mass and can be found in table 2 of ref. [44].

- $m_{H}$ and $m_{A}$. The $Z$ produced in the $A$ decay typically has a higher $p_{T}$ than the $Z$ 's produced in $H$ decay. Therefore we assume that the lower $p_{T} Z$ 's are coming from the $H$. For the reconstructed $H$ with mass $m_{Z Z}$ and $A$ with mass $m_{Z Z Z}$ we require:

$$
\begin{gathered}
0.9 m_{H}<m_{Z Z}<1.1 m_{H} \\
0.875 m_{A}<m_{Z Z Z}<1.125 m_{A} .
\end{gathered}
$$




\begin{tabular}{|l|cc|ccc|}
\hline Cut & $m_{H}=126 \mathrm{GeV}$ & $m_{H}=200 \mathrm{GeV}$ & BG [fb] & $S / B$ & $S / \sqrt{B}$ \\
\hline Leptonic decay & 1.0 & 10 & & & - \\
Four leptons, two jets [eq. (4.11)] & 0.14 & 2.78 & 2.592 & 1.07 & 29.9 \\
$Z$-mass [eq. (4.12)] & 0.027 & 1.03 & 0.6027 & 1.71 & 23.1 \\
$m_{Z Z}$ [eq. (4.13)] & 0.012 & 0.73 & 0.2118 & 3.49 & 27.9 \\
$m_{Z Z Z}$ [eq. (4.14)] & 0.0094 & 0.54 & 0.0905 & 5.98 & 31.2 \\
\hline
\end{tabular}

Table 3. Signal and background cross sections with cuts for signal benchmark point of $m_{A}=$ $400 \mathrm{GeV}$ and $m_{H}=126$ or $200 \mathrm{GeV}$ at the $14 \mathrm{TeV}$ LHC. We have chosen a nominal value for $\sigma \times \mathrm{BR}\left(g g \rightarrow A \rightarrow H Z \rightarrow Z Z Z \rightarrow 4 \ell+2 j\right.$ ) of $1.0 \mathrm{fb}$ (for $126 \mathrm{GeV} m_{H}$ ) and $10 \mathrm{fb}$ (for $200 \mathrm{GeV}$ $\left.m_{H}\right)$ to illustrate the cut efficiencies for the signal process. The total background cross section after cuts is shown by imposing the cuts for the $m_{H}=200 \mathrm{GeV}$ case. $S / B$ and $S / \sqrt{B}$ are given for the $m_{H}=200 \mathrm{GeV}$ benchmark point. In the last column, $S / \sqrt{B}$ is shown for an integrated luminosity of $\mathcal{L}=300 \mathrm{fb}^{-1}$.

In table 3 , we show the cross sections after cuts for two signal benchmark points $m_{H}=126 \mathrm{GeV}$ and $200 \mathrm{GeV}$ with $m_{A}$ fixed at $400 \mathrm{GeV}$, as well as for the SM backgrounds. For $m_{H}=126 \mathrm{GeV}$, we choose a signal cross section of $1 \mathrm{fb} .{ }^{4}$ For $m_{H}=200 \mathrm{GeV}$, we use a cross section of $10 \mathrm{fb}$ assuming $\operatorname{BR}(H \rightarrow Z Z)$ is $25 \%$ for $m_{H}=200 \mathrm{GeV}$. For the $m_{H}=126 \mathrm{GeV}$ case, due to the off-shell $Z$ decay, the cut efficiencies for identifying four leptons and two jets, as well as reconstructed $m_{Z Z}$ cuts are fairly low. Coupled with the small branching fraction of $H \rightarrow Z Z^{*}$, the number of surviving events is about 1 for $100 \mathrm{fb}^{-1}$ after all cuts are imposed. However, this channel becomes quite promising for heavier daughter masses when all $Z$ 's in the final state are on-shell, as shown for the benchmark point of $m_{H}=200 \mathrm{GeV}$.

We note that the nominal value for the cross section that is used in table 3 can, in typical BSM scenarios, be enhanced at small $\tan \beta$, due to the top loop contributions to the gluon fusion production, as well as the suppression of the $H \rightarrow b b$ branching fraction.

Figure 7 shows the $95 \%$ C.L. exclusion and $5 \sigma$ discovery at the $14 \mathrm{TeV}$ LHC for different integrated luminosities: $\mathcal{L}=100 \mathrm{fb}^{-1}, 300 \mathrm{fb}^{-1}$, and $1000 \mathrm{fb}^{-1}$. Even for $\mathcal{L}=300 \mathrm{fb}^{-1}$, the discovery limits vary only between about $3 \mathrm{fb}$ and $1.5 \mathrm{fb}$ with $200 \mathrm{GeV} m_{H}$ for $m_{A}$ between $300 \mathrm{GeV}$ and $600 \mathrm{GeV}$. Thus, the only challenge in this channel is to have high enough signal cross sections, as the SM backgrounds prove to be less of a threat compared to the bbll final state.

\section{Implications for the Type II 2HDM}

The decays $A / H \rightarrow H Z / A Z$ appear in many models that have an extension of the SM Higgs sector. In this section, we illustrate the implications of the exclusion or discovery limits of $b b \ell \ell, \tau \tau \ell \ell$ and $Z Z Z(4 \ell 2 j)$ searches on these models using Type II $2 \mathrm{HDM}$ as an explicit example.

\footnotetext{
${ }^{4}$ Particularly, the number is arrived at by taking gluon fusion cross section of $9 \mathrm{pb}$ for a $400 \mathrm{GeV}$ CP-odd Higgs, and assuming $\operatorname{BR}(A \rightarrow H Z)=50 \%$ and $\operatorname{Br}\left(H \rightarrow Z Z^{*}\right)=2.64 \%$ for a $126 \mathrm{GeV}$ Higgs.
} 

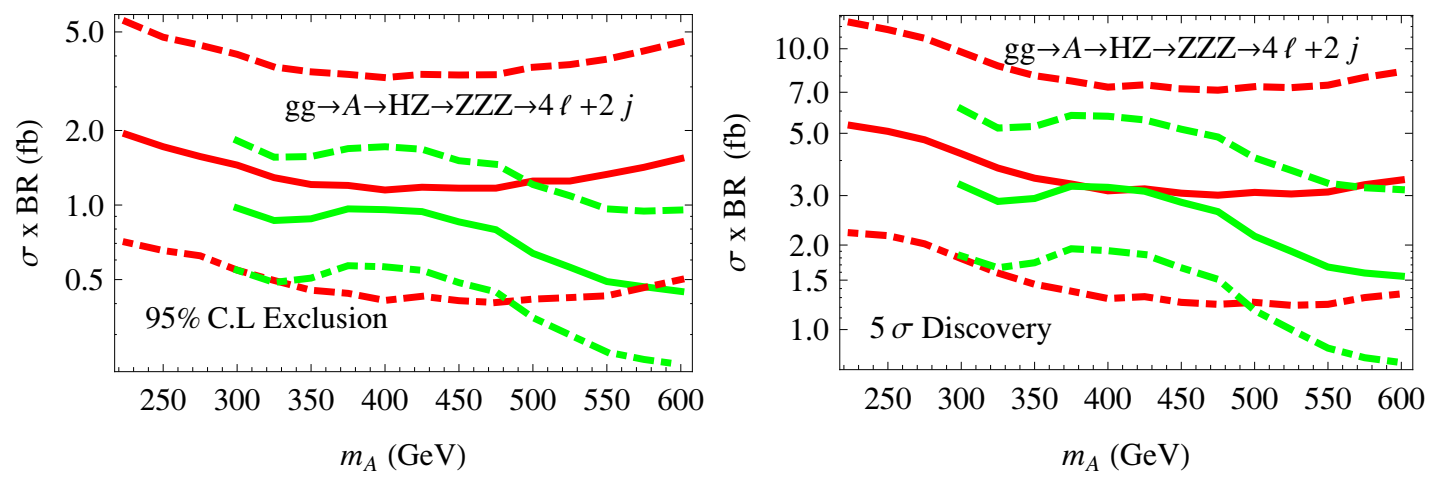

Figure 7. The $95 \%$ C.L. discovery and $5 \sigma$ exclusion limits at the $14 \mathrm{TeV}$ LHC in the channel $g g \rightarrow A \rightarrow H Z \rightarrow Z Z Z \rightarrow 4 \ell+2 j$ for $m_{H}=126 \mathrm{GeV}$ (red) and $m_{H}=200 \mathrm{GeV}$ (green). The dashed, solid and dot-dashed lines correspond to an integrated luminosity of 100, 300 and $1000 \mathrm{fb}^{-1}$, respectively. A $10 \%$ systematic error on the backgrounds is assumed as well.

\begin{tabular}{|l|l||l|l||l|l|}
\hline$\xi_{h^{0}}^{V V}$ & $\sin (\beta-\alpha)$ & $\xi_{H^{0}}^{V V}$ & $\cos (\beta-\alpha)$ & $\xi_{A}^{V V}$ & 0 \\
\hline$\xi_{h^{0}}^{u}$ & $\cos \alpha / \sin \beta$ & $\xi_{H^{0}}^{u}$ & $\sin \alpha / \sin \beta$ & $\xi_{A}^{u}$ & $\cot \beta$ \\
\hline$\xi_{h^{0}}^{d, l}$ & $-\sin \alpha / \cos \beta$ & $\xi_{H^{0}}^{d, l}$ & $\cos \alpha / \cos \beta$ & $\xi_{A}^{d, l}$ & $\tan \beta$ \\
\hline
\end{tabular}

Table 4. The multiplicative factors $\xi$ by which the couplings of the CP-even Higgses and the CPodd Higgs to the gauge bosons and fermions scale with respect to the SM value. The superscripts $u, d, l$ and $V V$ refer to the up-type quarks, down-type quarks, leptons, and $W W / Z Z$ respectively.

In the Type II $2 \mathrm{HDM}$, one Higgs doublet $\Phi_{1}$ provides masses for the down-type quarks and charged leptons, while the other Higgs doublet $\Phi_{2}$ provides masses for the up-type quarks. The couplings of the CP-even Higgses $h^{0}, H^{0}$ and the CP-odd Higgs $A$ to the SM gauge bosons and fermions are scaled by a factor $\xi$ relative to the SM value, which are presented in table 4.

The implication of the current Higgs search results on the Type II 2HDM has been studied in the literature $[15,16,18,23,45-48]$. In particular, a detailed analysis of the surviving regions of the Type II $2 \mathrm{HDM}$ was performed in [23], considering various theoretical constraints and including the latest experimental results from both the ATLAS and the CMS. Either the light or the heavy CP-even Higgs can be interpreted as the observed $126 \mathrm{GeV}$ SM-like Higgs, with very different preferred parameter regions. In the $h^{0}-126$ case, we are restricted to narrow regions with $\sin (\beta-\alpha) \sim \pm 1$ with $\tan \beta$ up to 4 or an extended region in $0.55<\sin (\beta-\alpha)<0.9$ with $1.5<\tan \beta<4$. The masses $m_{H^{0}}, m_{H^{ \pm}}$, and $m_{A}$ are, however, relatively unconstrained. In the $H^{0}-126$ case, we are restricted to a narrow region of $\sin (\beta-\alpha) \sim 0$ with $\tan \beta$ up to about 8 , or an extended region of $\sin (\beta-\alpha)$ between -0.8 to -0.05 , with $\tan \beta$ extending to 30 or higher. $m_{A}$ and $m_{H^{ \pm}}$ are nearly degenerate due to $\Delta \rho$ constraints. Imposing the flavor constraints in addition further narrows down the preferred parameter space.

Given the different parameter dependence of the gluon fusion cross section for $A$ and $H^{0}$, the branching fractions of $h^{0}, H^{0}$ and $A$, as well as the coupling difference between 


\begin{tabular}{|c|c|c|c|c|}
\hline$\left\{m_{A}, m_{H^{0}}, m_{h^{0}}\right\} \mathrm{GeV}$ & $A \rightarrow h^{0} Z$ & $A \rightarrow H^{0} Z$ & $H^{0} \rightarrow A Z$ & Favored region \\
\hline BP1: $\{400,126,50\}$ & $\checkmark$ & $\checkmark$ & $x$ & $\sin (\beta-\alpha) \approx 0$ \\
\hline BP2: $\{400,200,126\}$ & $\checkmark$ & $\checkmark$ & $x$ & $\sin (\beta-\alpha) \approx \pm 1$ \\
\hline BP3: $\{300,400,126\}$ & $\checkmark$ & $x$ & Marginal & $\sin (\beta-\alpha) \approx \pm 1$ \\
\hline BP4: $\{50,400,126\}$ & $x$ & $x$ & $\checkmark$ & $\sin (\beta-\alpha) \approx \pm 1$ \\
\hline BP5: $\{200,400,126\}$ & $x$ & $x$ & $\checkmark$ & $\sin (\beta-\alpha) \approx \pm 1$ \\
\hline
\end{tabular}

Table 5. Benchmark points shown for illustrating the discovery and exclusion limits in the processes considered in the context of the Type II 2HDM. The checkmarks indicate kinematically allowed channels. Also shown are the typical favored region of $\sin (\beta-\alpha)$ for each case (see ref. [23]).

$h^{0} A Z$ and $H^{0} A Z$, we can identify three different classes of processes: $g g \rightarrow A \rightarrow h^{0} Z$, $g g \rightarrow A \rightarrow H^{0} Z$, and $g g \rightarrow H^{0} \rightarrow A Z$ when interpreting the exclusion and discovery limits from the previous sections. We do not consider the decay of $h^{0} \rightarrow A Z$ since this channel is experimentally challenging given that both $h^{0}$ and $A$ are relatively light.

In table 5, we list the benchmark points that we use for the interpretation of the exclusion and discovery bounds in the Type II 2HDM. BP1 is the only $H^{0}-126$ case while BP2-BP5 are for the $h^{0}-126$ case. Both BP1 with $\left(m_{A}, m_{H^{0}}, m_{h^{0}}\right)=(400,126,50) \mathrm{GeV}$ and BP2 with $\left(m_{A}, m_{H^{0}}, m_{h^{0}}\right)=(400,200,126) \mathrm{GeV}$ are designed for both $g g \rightarrow A \rightarrow H^{0} Z$ and $g g \rightarrow A \rightarrow h^{0} Z$ as both modes are kinematically open. BP2 with $m_{H^{0}}=200 \mathrm{GeV}$, in particular, allow us to study the implication of $Z Z Z(4 \ell 2 j)$ search through $g g \rightarrow A \rightarrow H^{0} Z$. BP3 with $\left(m_{A}, m_{H^{0}}, m_{h^{0}}\right)=(300,400,126) \mathrm{GeV}$ is designed for $A \rightarrow h^{0} Z$ with the $H^{0}$ decoupled. We also choose $m_{A}$ to be below the $t \bar{t}$ threshold. BP4 with $\left(m_{A}, m_{H^{0}}, m_{h^{0}}\right)=$ $(50,400,126) \mathrm{GeV}$ and BP5 with $\left(m_{A}, m_{H^{0}}, m_{h^{0}}\right)=(200,400,126) \mathrm{GeV}$ are designed for the study of $g g \rightarrow H^{0} \rightarrow A Z$. Also shown in table 5 are the preferred regions in $\sin (\beta-\alpha)$ once all the theoretical and experimental constraints are imposed, following ref. [23].

Note that in our study, we have decoupled the charged Higgs so that it does not appear in the decay products of $A$ or $H$. For a light charged Higgs that is accessible in the decays of $A / H \rightarrow H^{ \pm} W^{\mp}, H^{+} H^{-}$, decay branching fractions of $A / H \rightarrow H Z / A Z$ will decrease correspondingly, which reduces the reach of this channel. However, the new decay channels involving the charged Higgs might provide new discovery modes for $A$ or $H$, which have been explored elsewhere [49-53]. In particular, for $A / H \rightarrow H^{ \pm} W^{\mp}, H^{+} H^{-}$ with $H^{ \pm} \rightarrow \tau^{ \pm} \nu$, the spin correlation in the $\tau$ decay can be used to identify the signal from the SM backgrounds. The sensitivity of this channel involving $H^{ \pm}$in the intermediate to large $\tan \beta$ region provides a nice complementarity to the $A / H \rightarrow H Z / A Z$ channels [49].

To be more general, in the discussion below when we interpret the search results of $b b \ell \ell, \tau \tau \ell \ell$ and $Z Z Z(4 \ell 2 j)$ channels in the model parameter space, we do not restrict ourselves to the narrow preferred parameter regions for $h^{0}-126$ or $H^{0}-126$ case as shown in ref. [23]. In particular, we consider the broad range of $-1 \leq \sin (\beta-\alpha) \leq+1$ and $1 \leq \tan \beta \leq 50$. This is because the allowed regions would change if a soft $\mathcal{Z}_{2}$ symmetry breaking is incorporated which ref. [23] did not deal with. Furthermore, the Higgs sector of $2 \mathrm{HDM}$ and the subsequent symmetry breaking structure is rather general and the results 

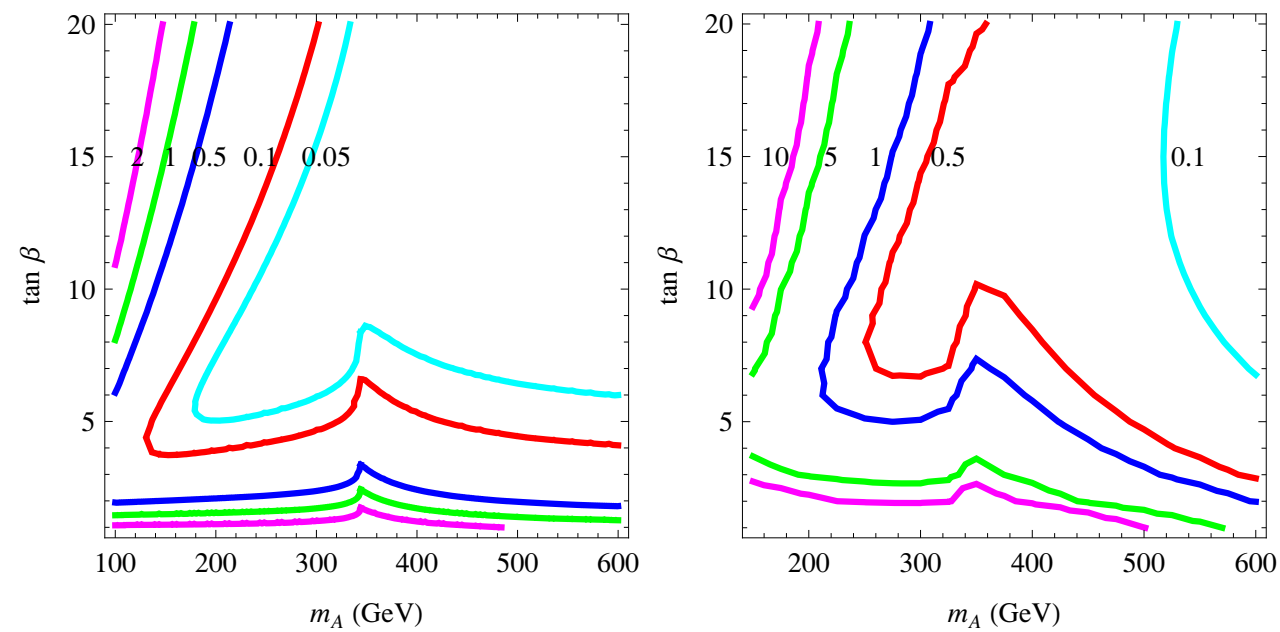

Figure 8. Contours of $\sigma(g g \rightarrow A)$ normalized to the SM value in the $m_{A}-\tan \beta$ plane (left panel) and $\sigma(g g \rightarrow A)$ at the $14 \mathrm{TeV}$ LHC in unit of pb (right panel).

presented in this section can be interpreted in the context of any such model if the Higgs couplings to the fermions follow a similar pattern. We do, however, point out the interplay between the exotic Higgs decay channels and the SM-like Higgs search results at the end of each discussion.

\section{$5.1 \quad g g \rightarrow A \rightarrow h^{0} Z$}

We compute the production cross section for the CP-odd Higgs $A$ by a simple rescaling of the SM Higgs cross section as follows:

$$
\sigma(g g \rightarrow A)=\sigma_{\mathrm{SM}} \times \frac{\left|\cot \beta F_{1 / 2}^{A}\left(\tau_{t}\right)+\tan \beta F_{1 / 2}^{A}\left(\tau_{b}\right)\right|^{2}}{\left|F_{1 / 2}^{h}\left(\tau_{t}\right)+F_{1 / 2}^{h}\left(\tau_{b}\right)\right|^{2}},
$$

where $\tau_{f}=4 m_{f}^{2} / m_{A}^{2}$ and the scalar and pseudoscalar loop factors $F_{1 / 2}^{h}$ and $F_{1 / 2}^{A}$ are given by $[22]$ :

$$
F_{1 / 2}^{A}=-2 \tau f(\tau), \quad F_{1 / 2}^{h}=-2 \tau[1+(1-\tau) f(\tau)]
$$

and

$$
f(\tau)= \begin{cases}{\left[\sin ^{-1}(1 / \sqrt{\tau})\right]^{2}} & \tau \geq 1 \\ -\frac{1}{4}\left[\ln \left(\eta_{+} / \eta_{-}\right)-i \pi\right]^{2} & \tau<1\end{cases}
$$

with $\eta_{ \pm} \equiv 1 \pm \sqrt{1-\tau}$. We have ignored the contribution from other Higgses in the loop, which is typically small. The left panel of figure 8 shows the contour plot of the $\sigma(g g \rightarrow A)$ normalized to that of the SM Higgs with the same mass. The $\tan \beta$ dependence is due to the Att and $A b b$ couplings, while the mass dependence comes from the different dependence of $F_{1 / 2}\left(\tau_{f}\right)$ on $\tau_{f}$ for pseudoscalar compared to a scalar. Enhancements over the SM value is possible for large $\tan \beta$ at small $m_{A}$ due to the bottom loop, or small $\tan \beta$ for all values of $m_{A}$ due to the top loop. The bump in the plot for $m_{A}$ around $350 \mathrm{GeV}$ corresponds to top threshold effects. Note that for $A$, the production cross section only depends on 

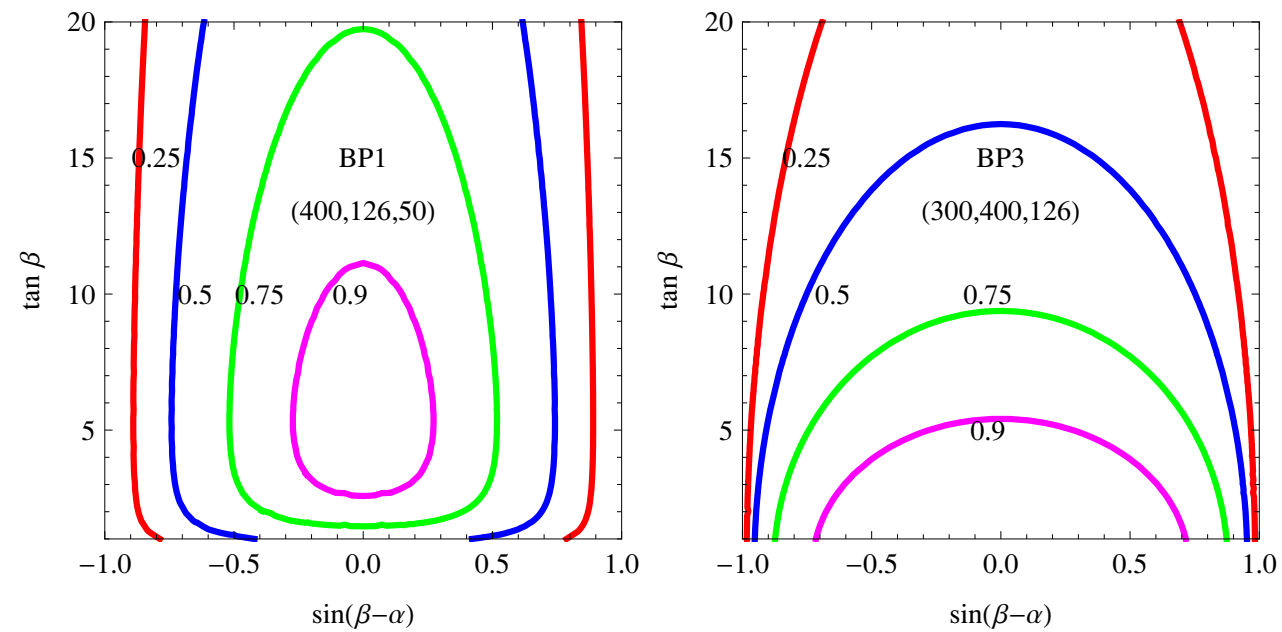

Figure 9. Contour plot of $\mathrm{BR}\left(A \rightarrow h^{0} Z\right)$ for BP1 (left panel), and BP3 (right panel). Also marked in each plot is the corresponding values of $\left(m_{A}, m_{H^{0}}, m_{h^{0}}\right)$ for each benchmark point.

$\tan \beta$ and is independent of $\alpha$. Also shown in the right panel of figure 8 are contours of $\sigma(g g \rightarrow A)$ in the $m_{A}-\tan \beta$ plane for the $14 \mathrm{TeV}$ LHC, with the cross sections for the SM Higgs production obtained from refs. [40, 54]. Significant cross sections of $10 \mathrm{pb}$ or more are possible for large $m_{A}$ up to $500 \mathrm{GeV}$ for small $\tan \beta$. Cross sections of similar magnitude are also possible at large $\tan \beta$ due to the bottom loop enhancement effects, albeit only for relatively small $m_{A}$.

In figure 9, we show contour plots of $\mathrm{BR}\left(A \rightarrow h^{0} Z\right)$ for BP1 (left panel) and BP3 (right panel). $\operatorname{BR}\left(A \rightarrow h^{0} Z\right)$ always maximizes at $\sin (\beta-\alpha)=0$, and decreases for larger $|\sin (\beta-\alpha)|$, since $g_{Z A h^{0}} \sim \cos (\beta-\alpha)$. For BP1 with $\left(m_{A}, m_{H^{0}}, m_{h^{0}}\right)=(400,126,50) \mathrm{GeV}$, both $A \rightarrow h^{0} Z$ and $A \rightarrow H^{0} Z$ open, with the coupling of the latter process proportional to $\sin (\beta-\alpha)$. Therefore, $\operatorname{BR}\left(A \rightarrow h^{0} Z\right)$ decreases more rapidly when $|\sin (\beta-\alpha)|$ gets bigger. $\operatorname{BR}\left(A \rightarrow h^{0} Z\right)$ decreases at large $\tan \beta$ as $A \rightarrow b b$ becomes more and more important. For $m_{A}>2 m_{t}, A \rightarrow t t$ becomes competitive at low $\tan \beta$, which correspondingly reduces $\mathrm{BR}\left(A \rightarrow h^{0} Z\right)$ further in that region. For BP2 with $\left(m_{A}, m_{H^{0}}, m_{h^{0}}\right)=(400,200,126) \mathrm{GeV}$, the behavior of $\operatorname{BR}\left(A \rightarrow h^{0} Z\right)$ is very similar to that of $\mathrm{BP} 1$.

For BP3 with $\left(m_{A}, m_{H^{0}}, m_{h^{0}}\right)=(300,400,126) \mathrm{GeV}$, only $A \rightarrow h^{0} Z$ opens with no competitive process from $A \rightarrow H^{0} Z$ and $A \rightarrow t t$. Therefore, comparing to $\operatorname{BP} 1, \operatorname{BR}(A \rightarrow$ $\left.h^{0} Z\right)$ decreases much slower as $\sin (\beta-\alpha)$ approaches \pm 1 . $\mathrm{BR}\left(A \rightarrow h^{0} Z\right)$ is also maximized at smaller $\tan \beta$ due to both the absence of $A \rightarrow t t$ and the suppression of $A \rightarrow b b$.

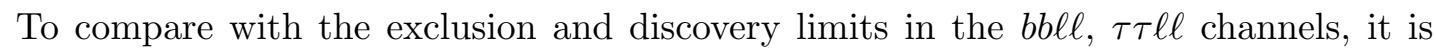
also important to know the branching fractions of $h^{0} \rightarrow b b, \tau \tau$, which depend mostly on $m_{h^{0}}$. For BP1 with $m_{h^{0}}=50 \mathrm{GeV}$, we used $\mathrm{BR}\left(h^{0} \rightarrow b b\right)=82 \%$ and $\mathrm{BR}\left(h^{0} \rightarrow \tau \tau\right)=8 \%$. For the other benchmark points with $h^{0}$ being the SM-like $126 \mathrm{GeV}$ Higgs, the branching fraction is obtained by rescaling the $\mathrm{SM}$ value of the $\mathrm{BR}$ with relevant coupling coefficients as given in table 4 . We show a contour plot of $\mathrm{BR}\left(h^{0} \rightarrow b b\right)$ in figure 10 for $h^{0}$ being the $126 \mathrm{GeV}$ Higgs. While $h^{0} \rightarrow b b$ reaches $80 \%$ and saturates in most of the parameter space, 


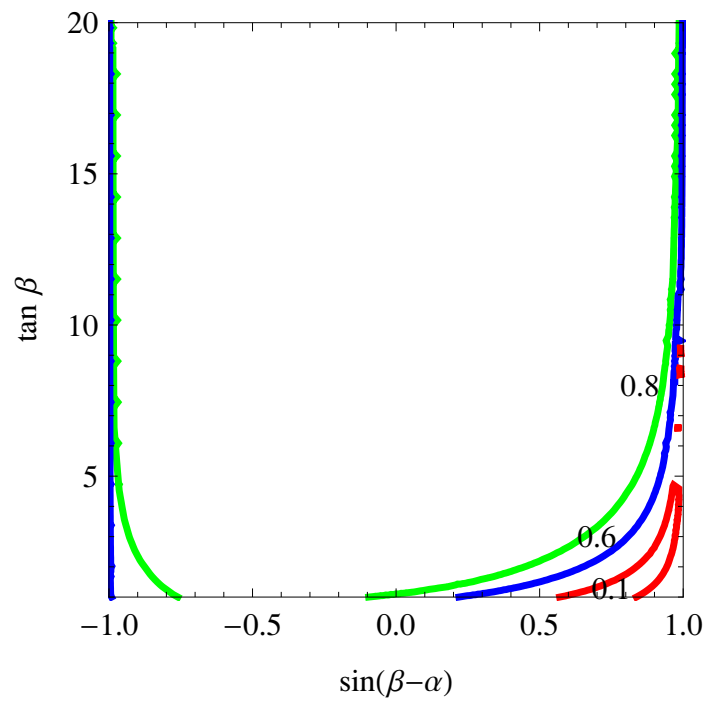

Figure 10. Branching ratio of $h^{0} \rightarrow b b$ for $h^{0}$ being the $126 \mathrm{GeV}$ Higgs.
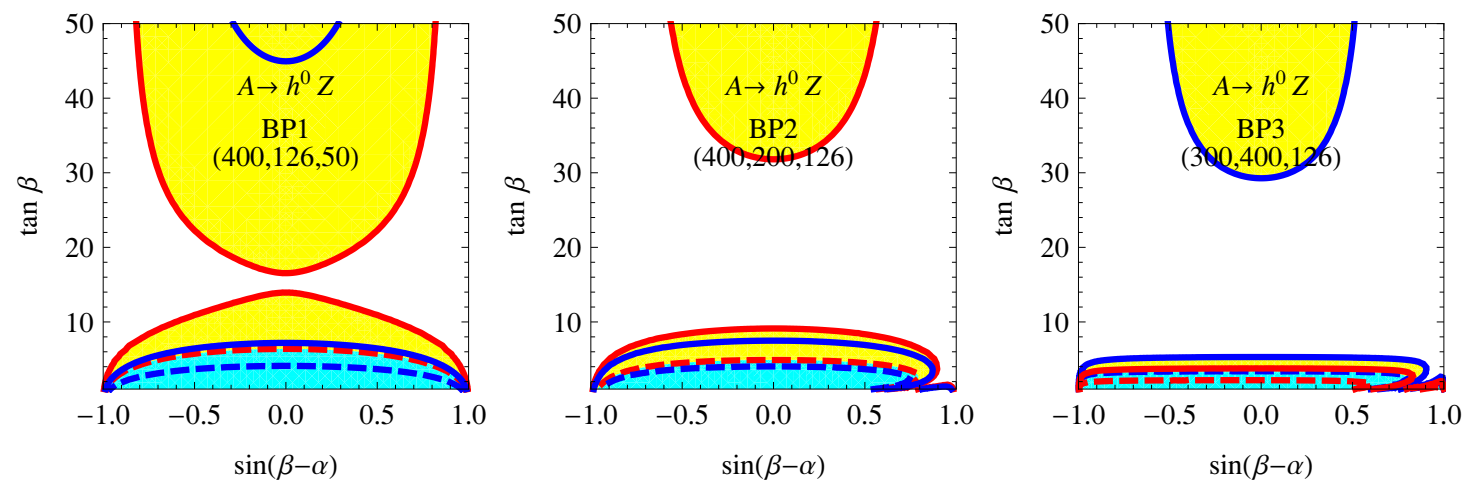

Figure 11. The $95 \%$ exclusion (yellow regions encoded by the solid lines) and $5 \sigma$ discovery (cyan regions enclosed by the dashed lines) for $g g \rightarrow A \rightarrow h^{0} Z$ in the $\tan \beta$ versus $\sin (\beta-\alpha)$ plane, corresponding to an integrated luminosity of $100 \mathrm{fb}^{-1}$ at the $14 \mathrm{TeV}$ LHC for BP1 (left panel), BP2 (middle panel) and BP3 (right panel). The red curves correspond to the bbll final state while the blue curves are the results for $\tau \tau \ell \ell$. Also marked in each plot is the corresponding values of $\left(m_{A}, m_{H^{0}}, m_{h^{0}}\right)$ for each benchmark point.

there is a wedge shaped region around $0.5<\sin (\beta-\alpha)<1$ at small $\tan \beta$ in which $h^{0} \rightarrow b b$ could be suppressed.

In figure 11, we show the LHC $100 \mathrm{fb}^{-1}$ discovery/exclusion reach for $g g \rightarrow A \rightarrow h^{0} Z$

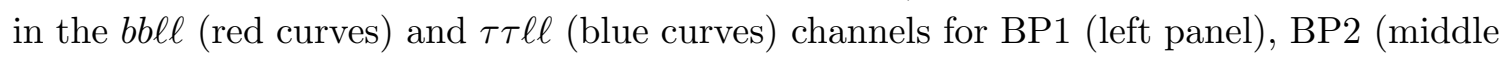
panel) and BP3 (right panel). 95\% Exclusion regions are shown as yellow regions enclosed by the solid lines while the $5 \sigma$ discovery regions are the cyan regions enclosed by the dashed lines. Each plot also indicates the corresponding values of $\left(m_{A}, m_{H^{0}}, m_{h^{0}}\right)$ for each specific benchmark point. For all the plots, the discovery region for either case is restricted to $\tan \beta \leq 5$ where the gluon-fusion cross section is enhanced from the toploop contribution. For BP1 with $m_{A}=400 \mathrm{GeV}$ and a small mass of $m_{h^{0}}=50 \mathrm{GeV}$, 

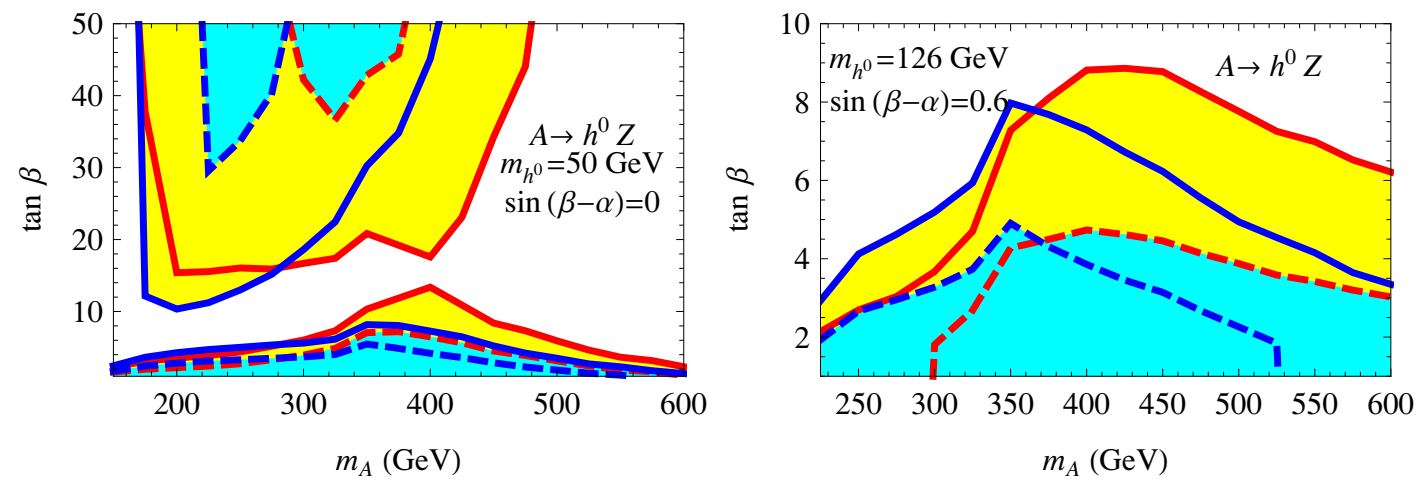

Figure 12. The $95 \%$ exclusion (yellow regions enclosed by the solid curves) and $5 \sigma$ discovery (cyan regions enclosed by dashed curves) in the $m_{A}-\tan \beta$ plane for $g g \rightarrow A \rightarrow h^{0} Z$ with $m_{h^{0}}=50 \mathrm{GeV}$, $\sin (\beta-\alpha)=0, m_{H^{0}}=126 \mathrm{GeV}$ (left panel) and $m_{h^{0}}=126 \mathrm{GeV}, \sin (\beta-\alpha)=0.6, m_{H^{0}}=1 \mathrm{TeV}$ (right panel), corresponding to an integrated luminosity of $100 \mathrm{fb}^{-1}$ at the $14 \mathrm{TeV}$ LHC. In either

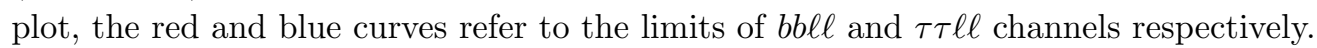

the experimental reach on $\sigma \times \mathrm{BR}$ is the best. Discovery is possible for all values of $-1<\sin (\beta-\alpha)<1$ for $\tan \beta$ up to 5 , while the exclusion region covers $\tan \beta \lesssim 14$ or large $\tan \beta \gtrsim 16$ with $-0.8<\sin (\beta-\alpha)<0.8$. Exclusion or discovery regions with $\tau \tau \ell \ell$ channel, shown in regions enclosed by the blue curves, are smaller compared to the regions in the bbll channel.

For BP2 with $m_{A}=400 \mathrm{GeV}$ and $m_{h^{0}}=126 \mathrm{GeV}$, regions of $\tan \beta<10$ or $\tan \beta>32$ will be excluded if no signal is detected, and regions of $\tan \beta<4$ can be discovered if there are positive signals. For BP3 with $m_{A}=300 \mathrm{GeV}$ and $m_{h^{0}}=126 \mathrm{GeV}$, the exclusion and discovery regions shrink further at small $\tan \beta$. The wedge-shaped region toward $\sin (\beta-\alpha)=1$ corresponds to the wedge region in figure 10 . Our results agree with that of ref. [21] for $A \rightarrow h^{0} Z$ with $h^{0}$ being the SM-like Higgs.

We note the interesting feature that the $b b \ell \ell$ limits are better than the $\tau \tau l \ell$ ones for BP1 and BP2, while the behavior flipped for BP3. This is because $\tau \tau \ell \ell$ typically has better reach than $b b \ell \ell$ process at small $m_{A}$, while $b b \ell \ell$ does better at large $m_{A}$, when the $\mathrm{BR}\left(h^{0} \rightarrow b b\right) / \mathrm{BR}\left(h^{0} \rightarrow \tau \tau\right) \sim 3 m_{b}^{2} / m_{\tau}^{2}$ is taken into account.

Given the smallness of the branching fraction of $h^{0} \rightarrow Z Z$ for the $m_{h^{0}}$ values chosen, the $Z Z Z$ channel will not be useful in probing the parameter space with $g g \rightarrow A \rightarrow h^{0} Z$. We also note that for the $H^{0}-126$ case (BP1) with the favored region to interpret $H^{0}$ as the SM-like Higgs being around $\sin (\beta-\alpha) \sim 0, g g \rightarrow A \rightarrow h^{0} Z$ will be extremely useful in probing this region. For the $h^{0}-126$ case (BP2 and BP3), the favored region to interpret $h^{0}$ as the SM-like Higgs is around $\sin (\beta-\alpha)= \pm 1$. Even though the $A \rightarrow h^{0} Z$ branching ratio is typically suppressed when $\sin (\beta-\alpha)$ approaches \pm 1 , we could still have reach in $\sin (\beta-\alpha)$ extending fairly close to \pm 1 .

In the left panel of figure 12, we show the reach in $\tan \beta$ versus $m_{A}$ plane for $m_{h^{0}}=$ $50 \mathrm{GeV}$ and $\sin (\beta-\alpha)=0$, with $95 \%$ C.L. exclusion (yellow regions enclosed by the solid curves) and $5 \sigma$ discovery (cyan regions enclosed by dashed curves) given for bbll channel (red lines) and $\tau \tau \ell \ell$ channel (blue lines). While $\tau \tau \ell \ell$ is more sensitive at low $m_{A}$, bbll 

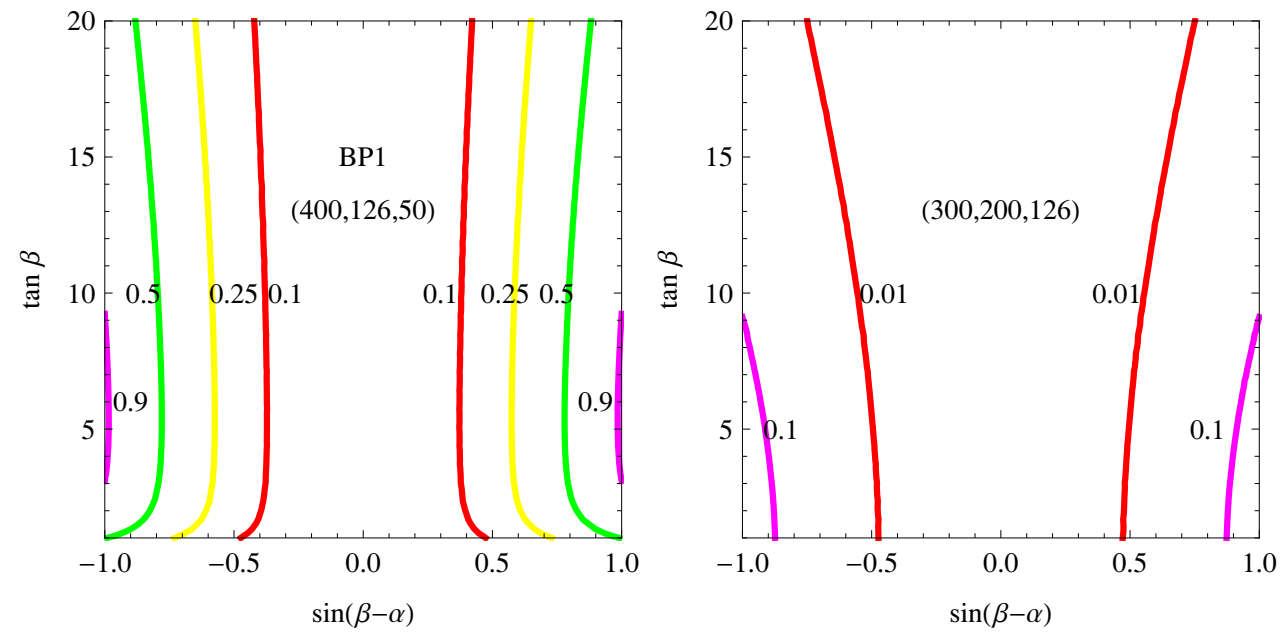

Figure 13. Contour plot of $\operatorname{BR}\left(A \rightarrow H^{0} Z\right)$ for $\mathrm{BP} 1$ (left panel) and a comparison point of $\left(m_{A}, m_{H^{0}}, m_{h^{0}}\right)=(300,200,126) \mathrm{GeV}$ (right panel).

extends the reach at large $m_{A}$. In general, small $\tan \beta$ (lower region) or large $\tan \beta$ (top region) are within reach due to the enhancement of the top and bottom Yukawa couplings in those regions. For small $\tan \beta \sim 1$, almost all values of $m_{A}$ up to $600 \mathrm{GeV}$ can be covered, with regions of $m_{A}$ shrink for increasing $\tan \beta$. At large $\tan \beta \gtrsim 10$, small $m_{A}$ can not be approached due to the weakening of the experimental limit, while large $m_{A}$ can not be approached due to the decreasing of the signal cross sections.

In the right panel of figure 12 , we show the reach in $m_{A}-\tan \beta$ plane for $m_{h^{0}}=126 \mathrm{GeV}$ and $\sin (\beta-\alpha)=0.6$. Note that we have chose a value for $\sin (\beta-\alpha)$ that is consistent with the current Higgs search results [23] of a $126 \mathrm{GeV} h^{0}$ while still allowing a sizable branching fraction for $A \rightarrow h^{0} Z$. We have decoupled the heavy CP-even Higgs $H^{0}$ so that $A \rightarrow H^{0} Z$ does not occur. Given the reduced branching fraction for $A \rightarrow h^{0} Z$, as well as the worse exclusion/discovery limits, the exclusion and discovery regions are smaller, compared to the left panel with $m_{h^{0}}=50 \mathrm{GeV}, \sin (\beta-\alpha)=0$. In particular, only regions with $\tan \beta \lesssim 8$ or a small region in $\tan \beta \gtrsim 50$ around $m_{A} \sim 450 \mathrm{GeV}$ are viable.

\section{$5.2 \quad g g \rightarrow A \rightarrow H^{0} Z$}

$A \rightarrow H^{0} Z$ opens once it is kinematically accessible. Since $m_{h^{0}}<m_{H^{0}}, A \rightarrow h^{0} Z$ is always accessible and more favorable in phase space. Whether $A \rightarrow H^{0} Z$ dominates or not depends largely on $\sin (\beta-\alpha)$, which controls the coupling of $Z A H^{0}$ as well as $Z A h^{0}$. Figure 13 shows the contours of $\operatorname{BR}\left(A \rightarrow H^{0} Z\right)$ in the parameter space of $\tan \beta$ versus $\sin (\beta-\alpha)$, for $\mathrm{BP} 1$ in the left panel. Contrary to the $A \rightarrow h^{0} Z$ case as shown in figure 9 , the branching ratios become larger for larger $|\sin (\beta-\alpha)|$, which is maximized at $\sin (\beta-\alpha)= \pm 1$, consistent with eq. (2.3). While the branching fractions are largely independent of $\tan \beta$, for small $\tan \beta \lesssim 2, \operatorname{BR}\left(A \rightarrow H^{0} Z\right)$ decreases due to the competition from $A \rightarrow t t$. The behavior of $\operatorname{BR}\left(A \rightarrow H^{0} Z\right)$ in $\mathrm{BP} 2$ with $\left(m_{A}, m_{H^{0}}, m_{h^{0}}\right)=(400,200,126) \mathrm{GeV}$ is very similar to that of BP1 with $\left(m_{A}, m_{H^{0}}, m_{h^{0}}\right)=(400,126,50) \mathrm{GeV}$. The branching fraction is slightly smaller compared to that of BP1 due to the relatively larger phase space suppression of 

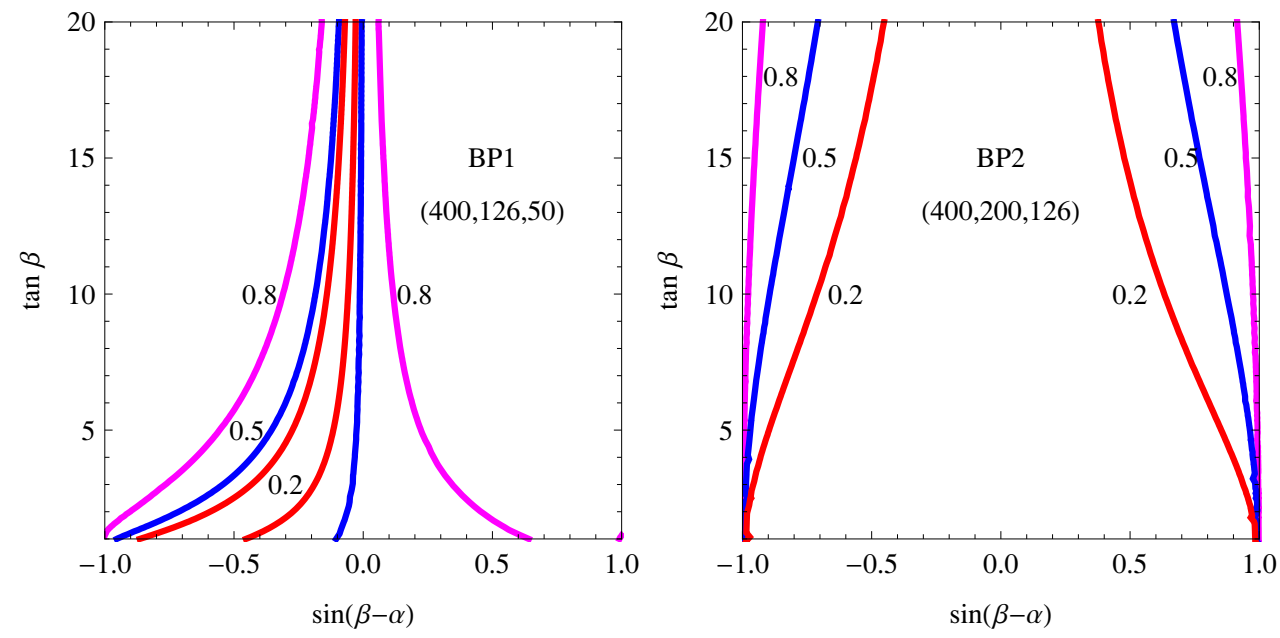

Figure 14. Contour plots of $\mathrm{BR}\left(H^{0} \rightarrow b b\right)$ for BP1 (left panel) and BP2 (right panel).

$A \rightarrow H^{0} Z$. As a comparison of the phase space effects, we show $\operatorname{BR}\left(A \rightarrow H^{0} Z\right)$ for $\left(m_{A}, m_{H^{0}}, m_{h^{0}}\right)=(300,200,126) \mathrm{GeV}$ in the right panel. The branching fraction is less than $10 \%$ over almost the entire parameter space. It is also evident that unlike BP1 and $\mathrm{BP} 2$, there is no suppression of the branching fractions at small $\tan \beta$ due to the absence of the $t t$ decay mode.

In figure 14, we show contours of the branching ratio $H^{0} \rightarrow b b$ for BP1 (left panel) and BP2 (right panel) in $\tan \beta$ versus $\sin (\beta-\alpha)$ plane. For BP1 with $m_{H^{0}}=126 \mathrm{GeV}$, $H^{0} \rightarrow b b$ is more than $80 \%$ for $\sin (\beta-\alpha)>0.1$ or $\sin (\beta-\alpha)<-0.2$ for large $\tan \beta$. The branching fraction decreases for smaller $\tan \beta$ due to the reduction of the bottom Yukawa coupling. The further reduction of the branching fraction in negative $\sin (\beta-\alpha)$ is due to the scaling of $H^{0} b b$ coupling as $\cos \alpha / \cos \beta$. For BP2 with $m_{H}=200 \mathrm{GeV}, H^{0} \rightarrow V V$ is kinematically accessible, which reduces $H^{0} \rightarrow b b$ further for small $\sin (\beta-\alpha)$. Note that for all the benchmark points chosen, $m_{H^{0}}<2 m_{t}$, and hence there is no suppression of the $b b$ mode for small $\tan \beta$ when the $t t$ mode would potentially dominate.

Figure 15 shows the exclusion reach (yellow regions enclosed by the solid lines) and discovery (cyan region enclosed by the dashed lines) of $A \rightarrow H^{0} Z$ for both the bbll (red) and $\tau \tau \ell \ell$ (blue) channels. Regions around $\sin (\beta-\alpha) \sim \pm 1$ are reachable while regions around $\sin (\beta-\alpha) \sim 0$ are inaccessible due to the suppression of $A \rightarrow H^{0} Z$. For BP1, $\tan \beta \lesssim 10$ can be excluded while $\tan \beta \lesssim 5$ is discoverable for $\sin (\beta-\alpha)= \pm 1$. The bottom loop effect kicks in at $\tan \beta \gtrsim 32$, excluding slices of parameter space around $\sin (\beta-\alpha)= \pm 1$. For $\tan \beta \sim 3,-1 \lesssim \sin (\beta-\alpha) \lesssim-0.5$ can be excluded, while for $\sin (\beta-\alpha)>0$, the exclusion reach extends to $\sin (\beta-\alpha) \gtrsim 0.2$ for small $\tan \beta$. There is also a small additional bump around $\sin (\beta-\alpha)=-0.6$, mainly due to the increasing of $\mathrm{BR}\left(H^{0} \rightarrow b b\right)$, as shown in the left panel of figure 14 . The reach is greatly reduced for BP2 due to the suppression of $H^{0} \rightarrow b b$, except for $\sin (\beta-\alpha) \sim \pm 1$. Only thin slices of parameter region near $\sin (\beta-\alpha) \sim \pm 1$ can be covered, which extends to $\tan \beta \lesssim 8$ for the exclusion, and $\tan \beta \lesssim 4.5$ for discovery. 

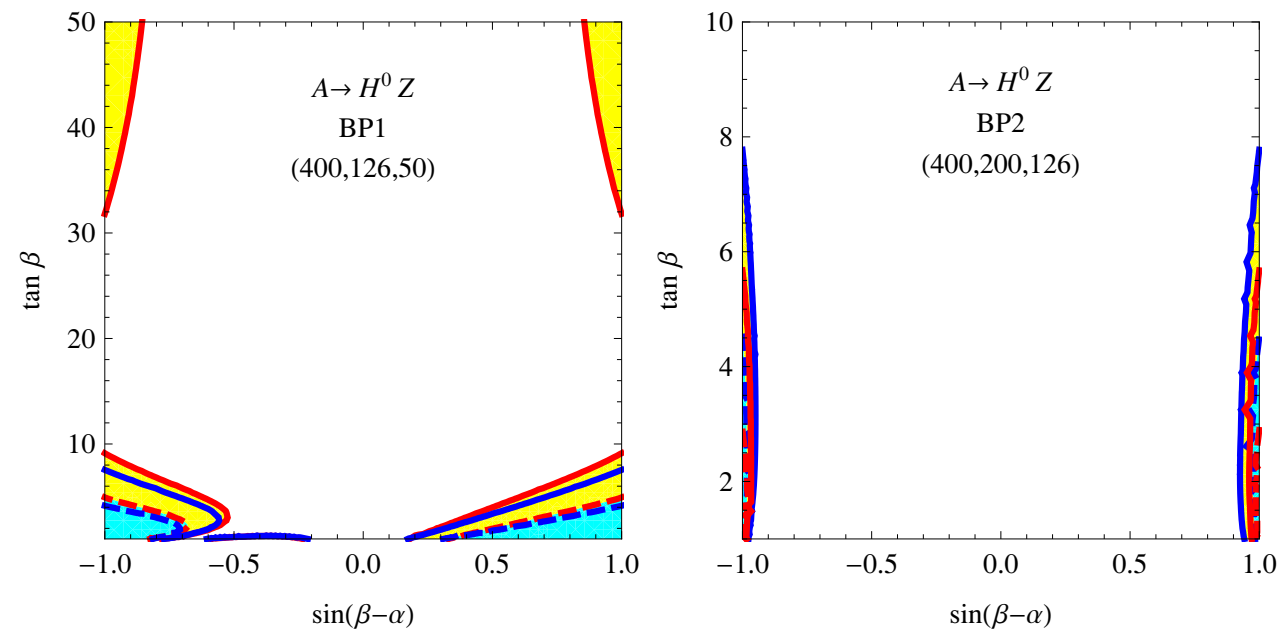

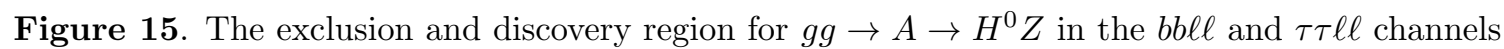
in the $\tan \beta$ versus $\sin (\beta-\alpha)$ plane, corresponding to an integrated luminosity of $100 \mathrm{fb}^{-1}$ for $\mathrm{BP} 1$ (left panel) and BP2 (right panel). Color coding is the same as in figure 11.

Note that for BP1 with $\left(m_{A}, m_{H^{0}}, m_{h^{0}}\right)=(400,126,50)$, both $A \rightarrow h^{0} Z$ and $A \rightarrow H^{0} Z$ open. The former is more sensitive to the $\sin (\beta-\alpha) \sim 0$ region, as shown in the left panel of figure 11, while the latter is more sensitive to $\sin (\beta-\alpha) \sim \pm 1$, as shown in the left panel of figure 15. Searches in these two channels are complementary to each other. When combined, they could cover the entire region of $\sin (\beta-\alpha)$, in particular, for $\tan \beta \lesssim 10$. Note that when combined with the current experimental search results for the $126 \mathrm{GeV}$ Higgs being the $H^{0}$, the region with $\sin (\beta-\alpha) \sim 0$ is favored, with a thin slice of extended region at negative $-0.8<\sin (\beta-\alpha)<-0.05$ as well [23].

Similar complementarity between $A \rightarrow h^{0} Z$ and $A \rightarrow H^{0} Z$ can be found for BP2 with $\left(m_{A}, m_{H^{0}}, m_{h^{0}}\right)=(400,200,126) \mathrm{GeV}$, for the entire region of $\sin (\beta-\alpha)$. Interpreting $h^{0}$ being the $126 \mathrm{GeV}$ observed Higgs boson, furthermore, favors $\sin (\beta-\alpha) \sim \pm 1$ or a thin slice of extended region at $0.55 \lesssim \sin (\beta-\alpha) \lesssim 0.9$ [23].

In the left panel of figure 16 , we present the exclusion and discovery reach in the $\tan \beta$ versus $m_{A}$ plane for $A \rightarrow H^{0} Z$ with $m_{H^{0}}=126 \mathrm{GeV}, m_{h^{0}}=50 \mathrm{GeV}$ and $\sin (\beta-\alpha)=-0.8$. We have chosen the value of $\sin (\beta-\alpha)$ such that the branching faction of $A \rightarrow H^{0} Z$ is sizable while still consistent with the experimental Higgs search results [23] with a $126 \mathrm{GeV}$ $H^{0}$. We see that $\tan \beta$ up to about 6.5 can be reached for exclusion, and $\tan \beta$ up to about 3.5 can be reached for discovery.

In the right panel of figure 16, we present the exclusion and discovery reach in the $\tan \beta$ versus $m_{A}$ plane for $m_{H}=200 \mathrm{GeV}, m_{h^{0}}=126 \mathrm{GeV}$ and $\sin (\beta-\alpha)=1$. For $350 \mathrm{GeV} \lesssim m_{A} \lesssim 600 \mathrm{GeV}, \tan \beta$ up to about 6 can be excluded, and up to about 3 can be discovered in the bbll channel. $\tau \tau \ell \ell$ channel does better in the low $m_{A}$ region.

For BP2 with $m_{A}=400 \mathrm{GeV}, m_{H^{0}}=200 \mathrm{GeV}$, we can also study the parameter reach of $A \rightarrow H^{0} Z$ with $H^{0} \rightarrow Z Z$. In figure 17 , we show $\mathrm{BR}\left(H^{0} \rightarrow Z Z\right)$ in the left panel, which reaches a maximum of $25 \%$ for $|\sin (\beta-\alpha)| \lesssim 0.2$. It gets larger for small $\tan \beta$ when $H^{0} \rightarrow b b$ is further suppressed. In the right panel of figure 17, we show the discovery and 

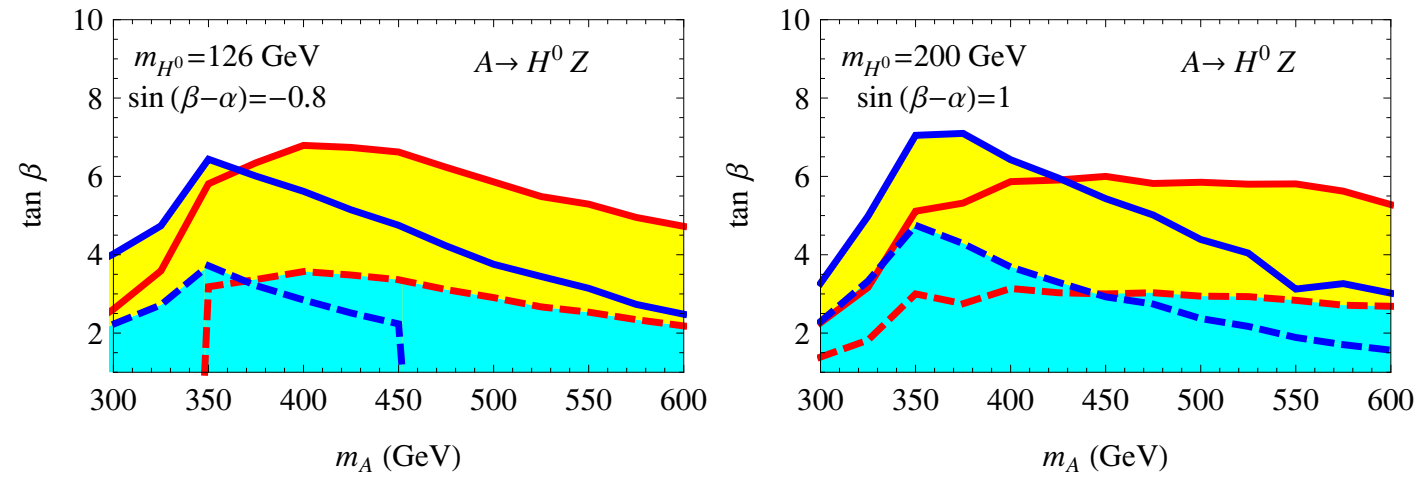

Figure 16. The discovery and exclusion regions in the $m_{A}-\tan \beta$ plane for $g g \rightarrow A \rightarrow H^{0} Z$ in bbll and $\tau \tau \ell \ell$ final states with $m_{H^{0}}=126 \mathrm{GeV}, m_{h^{0}}=50 \mathrm{GeV}, \sin (\beta-\alpha)=-0.8$ (left panel) and $m_{H^{0}}=200 \mathrm{GeV}, m_{h^{0}}=126 \mathrm{GeV}, \sin (\beta-\alpha)=1$ (right panel), corresponding to an integrated luminosity of $100 \mathrm{fb}^{-1}$ at the $14 \mathrm{TeV}$ LHC. Color coding is the same as in figure 12 .
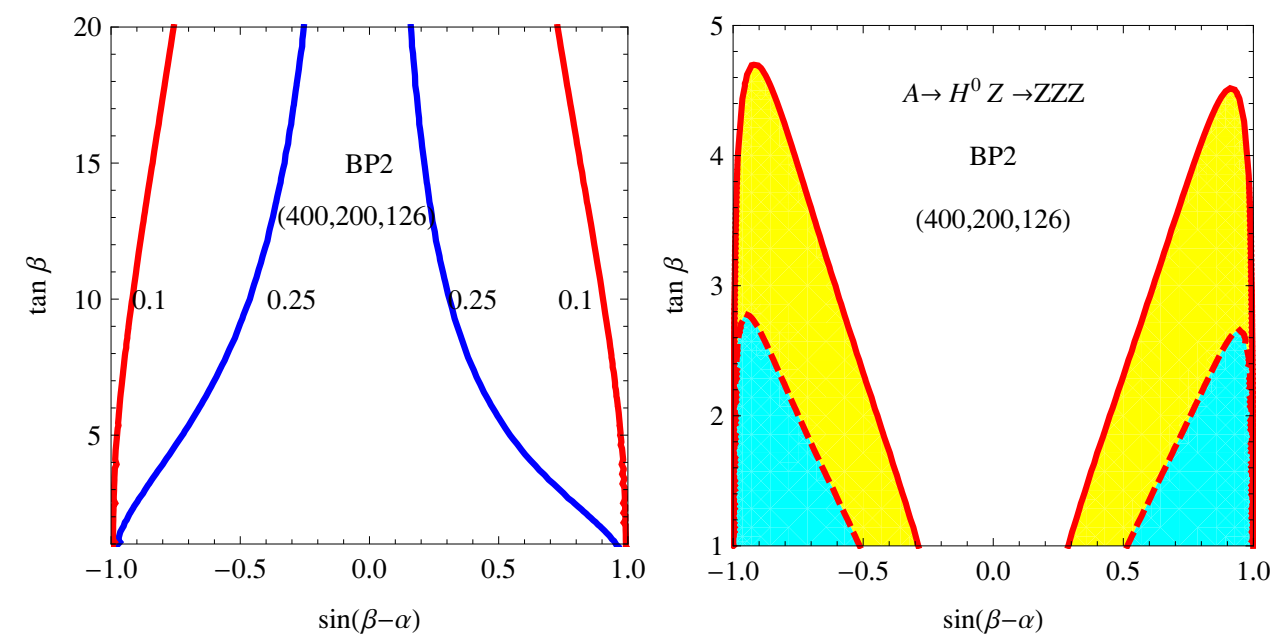

Figure 17. Left: contour plots of $\mathrm{BR}\left(H^{0} \rightarrow Z Z\right)$ for BP2. Right: the exclusion (yellow regions enclosed by the solid lines) and the discovery (cyan regions enclosed by the dashed lines) in the $\sin (\beta-\alpha)-\tan \beta$ plane for $g g \rightarrow A \rightarrow H^{0} Z \rightarrow Z Z Z$, corresponding to an integrated luminosity of $100 \mathrm{fb}^{-1}$ at the $14 \mathrm{TeV}$ LHC for the $4 \ell+2 j$ final state.

exclusion contours in the $\tan \beta$ versus $\sin (\beta-\alpha)$ plane for $100 \mathrm{fb}^{-1}$ luminosity at the LHC. While $H^{0} \rightarrow Z Z$ maximizes at $\sin (\beta-\alpha) \sim 0, A \rightarrow H^{0} Z$ is minimized in this region. As a result, regions of $0.3 \lesssim|\sin (\beta-\alpha)| \lesssim 1$ with $\tan \beta$ up to 4.7 can be excluded while the discovery regions are $0.5 \lesssim|\sin (\beta-\alpha)| \lesssim 1$ with $\tan \beta \lesssim 2.8$. Note also that this channel is complementary to $A \rightarrow H^{0} Z \rightarrow b b / \tau \tau \ell \ell$ as shown in figure 15 , which is sensitive to $\sin (\beta-\alpha) \sim \pm 1$ region.

In figure 18, we present the exclusion and discovery in $\tan \beta$ versus $m_{A}$ plane for $g g \rightarrow A \rightarrow H^{0} Z \rightarrow Z Z Z(4 \ell 2 j)$ with $m_{H}=200 \mathrm{GeV}, \sin (\beta-\alpha)=0.9$. We have chosen the value of $\sin (\beta-\alpha)$ such that the branching fractions of both $A \rightarrow H^{0} Z$ and $H^{0} \rightarrow Z Z$ is sizable while still consistent with the experimental Higgs search results [23] with a $126 \mathrm{GeV}$ $h^{0}$. We see that the whole region of $300 \mathrm{GeV}<m_{A}<600 \mathrm{GeV}$ can be covered at small 


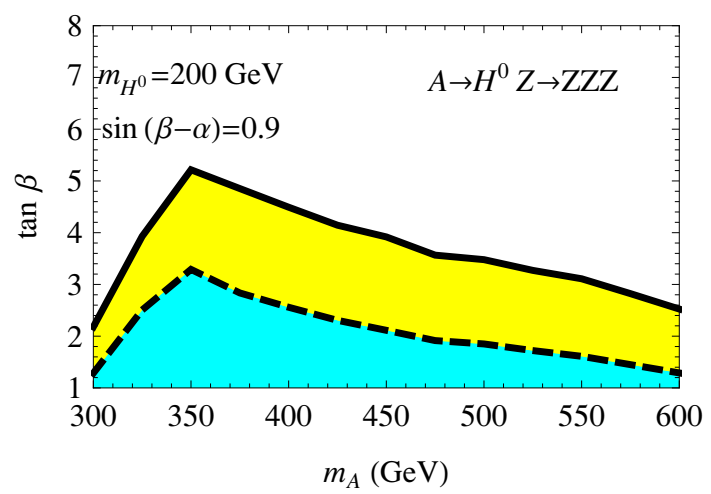

Figure 18. The exclusion (yellow region enclosed by the solid lines) and the discovery (cyan region enclosed by the dashed lines) in the $m_{A}-\tan \beta$ plane with $m_{H^{0}}=200 \mathrm{GeV}, m_{h^{0}}=126 \mathrm{GeV}$, and $\sin (\beta-\alpha)=0.9$, corresponding to an integrated luminosity of $100 \mathrm{fb}^{-1}$ at the $14 \mathrm{TeV} \mathrm{LHC}$ for the $4 \ell+2 j$ final state.

$\tan \beta$, with the maximum reach in $\tan \beta$ obtained for $m_{A} \sim 350 \mathrm{GeV}: \tan \beta \lesssim 3$ for discovery and $\tan \beta \lesssim 5$ for exclusion.

\section{$\mathbf{5 . 3} \quad g g \rightarrow H^{0} \rightarrow A Z$}

For this process, we restrict to the $m_{h^{0}}=126 \mathrm{GeV}$ case with a heavier $H^{0}$. We use BP4 with $\left(m_{A}, m_{H^{0}}, m_{h^{0}}\right)=(50,400,126) \mathrm{GeV}$ and BP5 with $\left(m_{A}, m_{H^{0}}, m_{h^{0}}\right)=(200,400,126) \mathrm{GeV}$ as an illustration. The gluon fusion production cross section for $H^{0}$ can be rescaled from the SM cross section:

$$
\sigma\left(g g \rightarrow H^{0}\right)=\sigma_{\mathrm{SM}} \times \frac{\left|\left(\frac{\sin \alpha}{\sin \beta}\right) F_{1 / 2}^{h}\left(\tau_{t}\right)+\left(\frac{\cos \alpha}{\cos \beta}\right) F_{1 / 2}^{h}\left(\tau_{b}\right)\right|^{2}}{\left|F_{1 / 2}^{h}\left(\tau_{t}\right)+F_{1 / 2}^{h}\left(\tau_{b}\right)\right|^{2}},
$$

where the loop factors $F$ 's are defined in eq. (5.2). We note that in contrast to the production of $A$ in eq. (5.1), the production of $H^{0}$ involves both $\alpha$ and $\beta$. In the left panel of figure 19, we show contours of the production cross section of $H^{0}$ normalized to the SM value in the $\sin (\beta-\alpha)-\tan \beta$ plane for $m_{H^{0}}=400 \mathrm{GeV}$. We see that for positive $\sin (\beta-\alpha)$, the cross section is always relatively more suppressed than that for negative $\sin (\beta-\alpha)$, introduced by the interference between the top and bottom loops in eq. (5.4). For $\sin (\beta-\alpha)= \pm 1$, which is preferred by the interpretation of $h^{0}$ being the SM-like Higgs, the cross section receives the strongest suppression: only $10 \%$ of the corresponding SM value. In the right panel of figure 19, we show contours of the production cross section at $14 \mathrm{TeV}$ LHC in the $m_{H^{0}}-\tan \beta$ plane. We see that cross sections of $10 \mathrm{pb}$ or more is possible for $m_{H^{0}}$ up to $425 \mathrm{GeV}$ for small $\tan \beta$ - slightly lower than the corresponding numbers for $\sigma(g g \rightarrow A)$ as shown in figure 8. However, the bottom loop enhancement plays a slightly more significant role in this case at large $\tan \beta$, compared to the $A$ case.

Figure 20 shows the $\mathrm{BR}\left(H^{0} \rightarrow A Z\right)$ for BP4 (left panel) and BP5 (right panel). Since $g_{Z A H^{0}} \propto \sin (\beta-\alpha)$, the branching fraction gets bigger for larger $|\sin (\beta-\alpha)|$, and is maximized at $\sin (\beta-\alpha)= \pm 1$. Branching fractions in BP4 is larger than that of BP5 due 

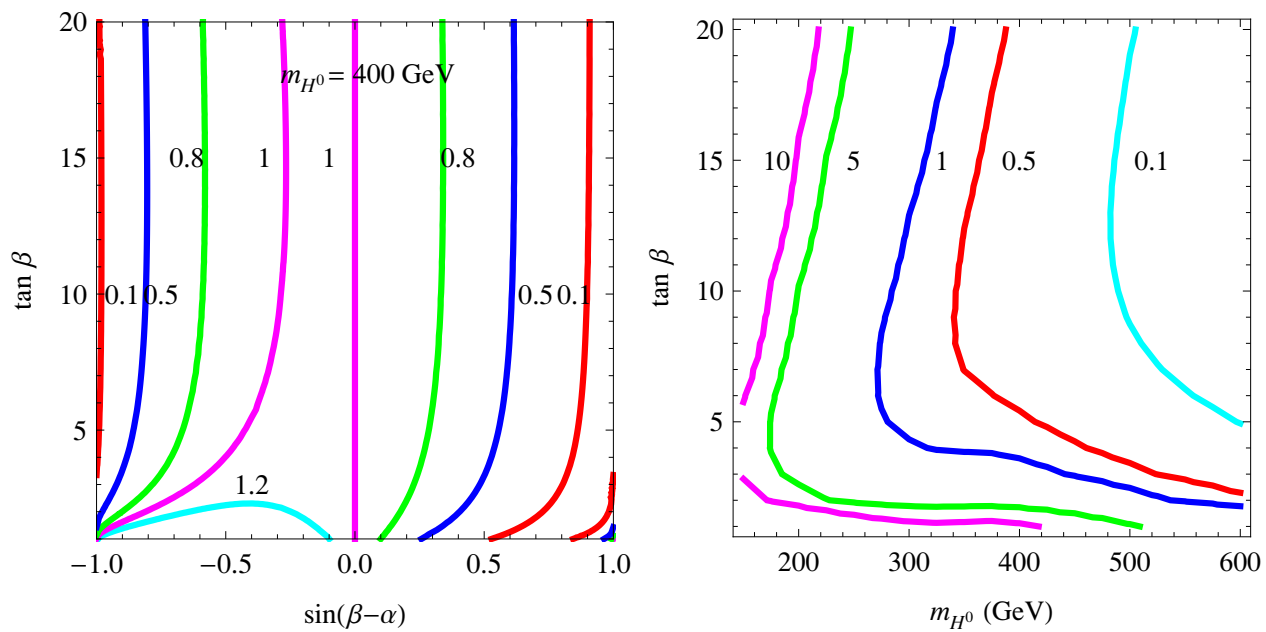

Figure 19. Contours of the cross section normalized to the SM value in the $\sin (\beta-\alpha)-\tan \beta$ plane (left panel) for $m_{H^{0}}=400 \mathrm{GeV}$ and $g g \rightarrow H^{0}$ cross section at the $14 \mathrm{TeV}$ LHC in unit of pb in the $m_{H^{0}}-\tan \beta$ plane (right panel) with $\sin (\beta-\alpha)=-1$.
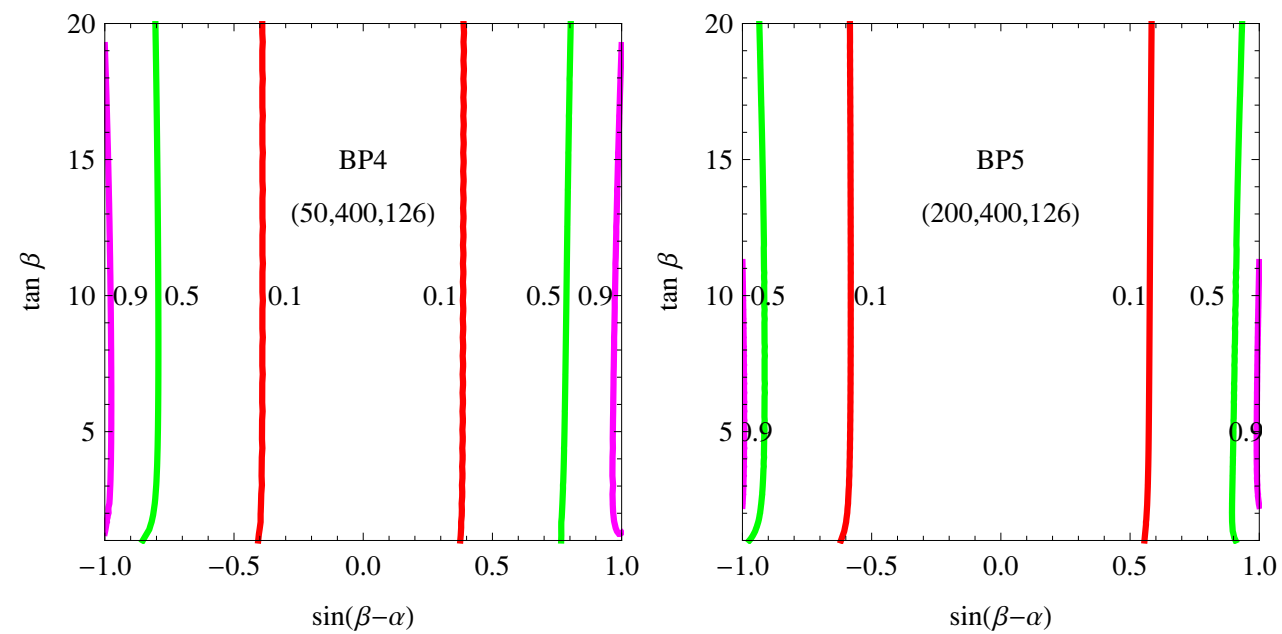

Figure 20. Contour plot of $\mathrm{BR}\left(H^{0} \rightarrow A Z\right)$ (left panel) for BP4 (left panel) and BP5 (right panel).

to the bigger phase space for $H^{0} \rightarrow A Z$. For $A \rightarrow b b$ and $\tau \tau$, the branching fraction is about $94 \%$ and $6 \%$ respectively, which does not vary much for BP4 with $m_{A}=50 \mathrm{GeV}$ and BP5 with $m_{A}=200 \mathrm{GeV}$.

In figure 21, we display the discovery/exclusion reach in $g g \rightarrow H^{0} \rightarrow A Z$ for the bbll (red) and $\tau \tau \ell \ell$ (blue) final states for BP4 (left panel) and BP5 (right panel). For BP4, large regions of parameter spaces in $\tan \beta$ versus $\sin (\beta-\alpha)$ can be excluded, except for $-0.15<\sin (\beta-\alpha)<0.2$ when $H^{0} \rightarrow A Z$ is highly suppressed. The discovery region shrinks to $-1 \lesssim \sin (\beta-\alpha) \lesssim-0.3$ and $0.35 \lesssim \sin (\beta-\alpha) \lesssim 0.9$ for all values of $\tan \beta$. For BP5, regions of $-1 \lesssim \sin (\beta-\alpha) \lesssim-0.5$ for all $\tan \beta$ and $0.6 \lesssim \sin (\beta-\alpha) \lesssim 0.8$ with $6 \lesssim \tan \beta \lesssim 26$ can be excluded and a smaller region in $-1 \lesssim \sin (\beta-\alpha) \lesssim-0.6$ with 

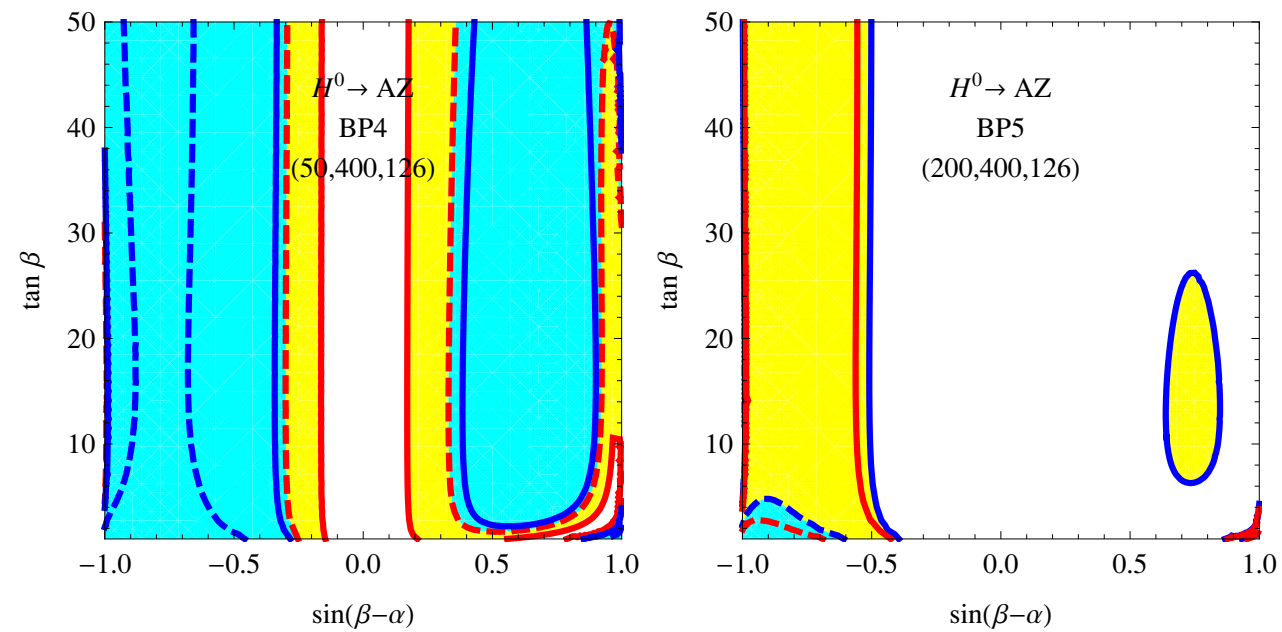

Figure 21. The exclusion and discovery regions in the $\tan \beta$ versus $\sin (\beta-\alpha)$ plane for $g g \rightarrow$ $H^{0} \rightarrow A Z$ with $b b \ell \ell$ (red) and $\tau \tau \ell \ell$ (blue) final states for BP4 (left panel) and BP5 (right panel), corresponding to $100 \mathrm{fb}^{-1}$ integrated luminosity at the $14 \mathrm{TeV}$ LHC. Color coding is the same as in figure 11.
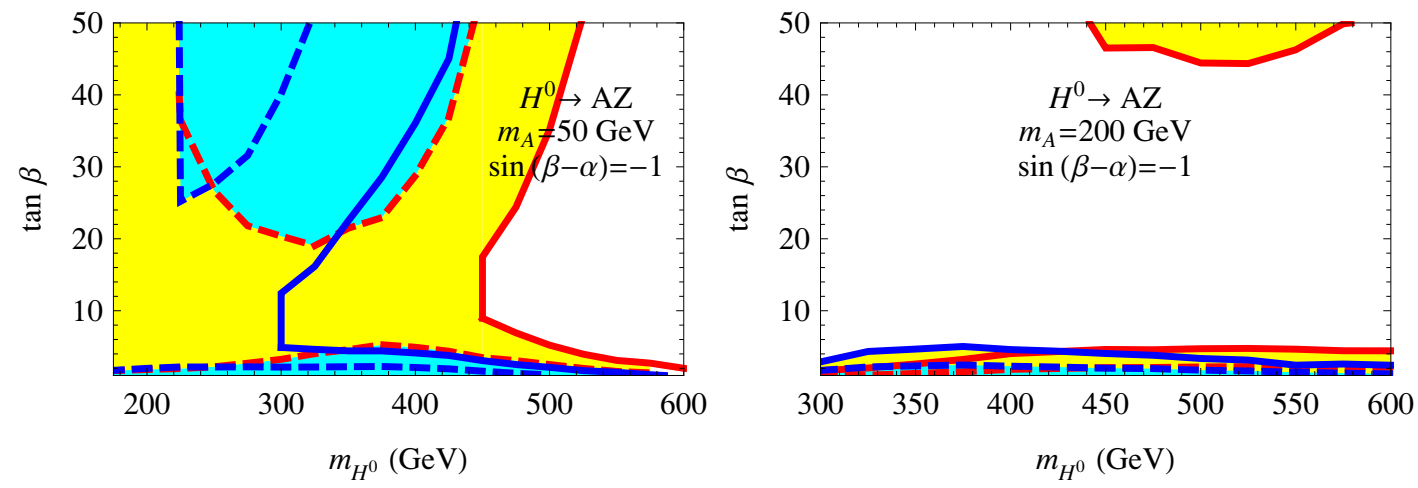

Figure 22. The discovery and exclusion region in the $m_{H^{0}}-\tan \beta$ plane for $g g \rightarrow H^{0} \rightarrow A Z$ with $b b \ell \ell$ (red) and $\tau \tau \ell \ell$ (blue) final states, corresponding to $100 \mathrm{fb}^{-1}$ integrated luminosity at the $14 \mathrm{TeV}$ LHC. The left panel is for $m_{A}=50 \mathrm{GeV}$ with $\sin (\beta-\alpha)=-1$ and the right panel is for $m_{A}=200 \mathrm{GeV}$ with $\sin (\beta-\alpha)=-1$. Color coding is the same as in figure 12 .

$\tan \beta \lesssim 5$ can be discovered. While bbll channel has better reach for BP $4, \tau \tau \ell \ell$ channel has a slightly better sensitivity for BP5. The reach is also much better for negative $\sin (\beta-\alpha)$ because of the less suppressed cross sections of $g g \rightarrow H^{0}$.

In the left panel of figure 22 , we show the exclusion and discovery each with $100 \mathrm{fb}^{-1}$ luminosity at $14 \mathrm{TeV}$ LHC in $\tan \beta$ versus $m_{H}$ plane, for $g g \rightarrow H^{0} \rightarrow A Z$ with bbll (red) and $\tau \tau \ell \ell$ (blue) final states. We have chosen $m_{A}=50 \mathrm{GeV}$ and $\sin (\beta-\alpha)=-1$. Discovery is possible for small values of $\tan \beta \lesssim 5$ or larger values of $\tan \beta \gtrsim 20$. The exclusion reach, however, is much more extended. All values of $\tan \beta$ can be covered for $m_{H^{0}}$ up to $450 \mathrm{GeV}$, with reach extended further at larger and smaller values of $\tan \beta$. The reach with daughter particle mass $m_{A}=200 \mathrm{GeV}$ is shown in the right panel of figure 22. Both the exclusion 
and discovery regions shrink greatly. Only very small $\tan \beta \lesssim 4$ or very large $\tan \beta \gtrsim 44$ can be excluded. Note that while $\sin (\beta-\alpha)= \pm 1$ is preferred by the interpretation of the $h^{0}$ being the SM-like Higgs, the suppression of $g g \rightarrow H^{0}$ in that region results in a reduced exclusion/discovery reach. Even a small deviation of $\sin (\beta-\alpha)$ away from \pm 1 would introduce a much larger reach in $g g \rightarrow H^{0} \rightarrow A Z$.

\section{Conclusion}

Given the discovery of a $126 \mathrm{GeV}$ SM-like Higgs boson at the LHC, it is now time to use the experimental data to constrain new physics models while also exploring the detectability of extra Higgs bosons in the extensions of the SM. In this spirit, we explored the production and decay of heavy scalar and pseudoscalar states via the processes $g g \rightarrow H^{0} \rightarrow A Z$ and $g g \rightarrow A \rightarrow h^{0} Z / H^{0} Z$ with both fermionic $(b b, \tau \tau)$ and possible bosonic $(Z Z)$ decays of the daughter Higgs. This channel provides nice complementarity to the conventional search channel $p p \rightarrow A / H \rightarrow \tau \tau$, which is mostly sensitive to the large $\tan \beta$ region. We presented model independent limits on the $95 \%$ C.L. exclusion and $5 \sigma$ discovery in those channels at the $14 \mathrm{TeV}$ LHC. The possibilities include the interesting case of having the $126 \mathrm{GeV}$ SM-like Higgs as a decay product of a heavy pseudoscalar.

For the $14 \mathrm{TeV}$ LHC with $300 \mathrm{fb}^{-1}$ integrated luminosity, the $95 \%$ C.L. limits on $\sigma \times \mathrm{BR}$ for the $b b l \ell$ final state (where the $b$ 's come from the Higgs in the final state) for a $126 \mathrm{GeV}$ daughter Higgs particle vary between $200 \mathrm{fb}$ to a few fb for the parent heavy Higgs mass in the range of $200 \mathrm{GeV}$ to $600 \mathrm{GeV}$, while the limit for $5 \sigma$ discovery is about $3-5$ times larger. For the $\tau \tau \ell \ell$ channel with the same range of $A$ mass, the exclusion bounds are around $5-1 \mathrm{fb}$ and the discovery reach is about $20 \mathrm{fb}-3 \mathrm{fb}$. While the $\sigma \times \mathrm{BR}$ reach in the $\tau \tau \ell \ell$ channel is in

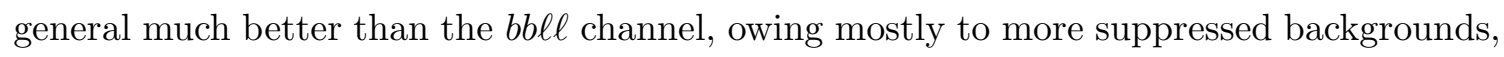
it is comparable to $b b \ell \ell$ mode once the branching fraction difference between $b b$ and $\tau \tau$ modes are taken into account in a given model. $g g \rightarrow A \rightarrow H^{0} Z \rightarrow Z Z Z \rightarrow 4 \ell 2 j$ is useful for heavy Higgses with $m_{H^{0}}>2 m_{Z}$. For $m_{H^{0}}=200 \mathrm{GeV}$ and $m_{A}=400 \mathrm{GeV}$, exclusion in this channel with $300 \mathrm{fb}^{-1}$ integrated luminosity requires as little as $1 \mathrm{fb}$ in $\sigma \times \mathrm{BR}$ while $5 \sigma$ discovery needs about $3 \mathrm{fb}$.

We then discussed the implication of the exclusion and discovery bounds of $b b \ell \ell, \tau \tau \ell \ell$ and $Z Z Z$ channels in the Type II $2 \mathrm{HDM}$, studying three classes of processes: $g g \rightarrow A \rightarrow$ $h^{0} Z, g g \rightarrow A \rightarrow H^{0} Z$, and $g g \rightarrow H^{0} \rightarrow A Z$. We find, in general, that there is a significant portion of the $\tan \beta$ versus $\sin (\beta-\alpha)$ plane that allows discovery/exclusion possibilities in the $b b \ell \ell$ and $\tau \tau \ell \ell$ final states. bbll and $\tau \tau \ell \ell$ have comparable reach, with $\tau \tau \ell \ell$ being

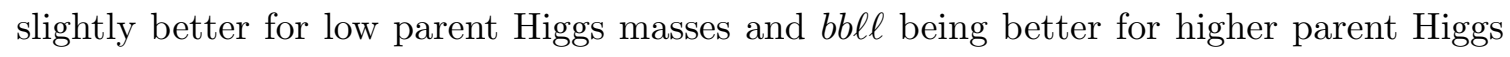
masses.

Specifically, in the channel $g g \rightarrow A \rightarrow h^{0} Z$ when $H^{0}$ is identified as the SM-like Higgs, $95 \%$ exclusion covers most of the $\tan \beta$ versus $\sin (\beta-\alpha)$ plane for $m_{A}$ around $400 \mathrm{GeV}$. $\tan \beta<5$ can also be covered by $5 \sigma$ discovery. On the other hand, the exclusion/discovery range is more restricted when $h^{0}$ is identified as the SM Higgs. Typically, we find that for $m_{A}=400 \mathrm{GeV}$, discovery region lies between $-1<\sin (\beta-\alpha) \lesssim 0.8$ and $\tan \beta \leq 5$, while the exclusion region extends to $\tan \beta \lesssim 10$ or $\gtrsim 30$. Note also that even though the reach 
is always maximized at $\sin (\beta-\alpha) \sim 0$, it extends to larger values of $|\sin (\beta-\alpha)|$ close to \pm 1 as well. A wide range of $m_{A}$ can be covered at low $\tan \beta \lesssim 10$, while high $\tan \beta$ can only be approached for $m_{A} \lesssim 500 \mathrm{GeV}$.

The case where $A$ decays to $H^{0} Z$ is complementary to $A \rightarrow h^{0} Z$ in that the discovery and exclusion regions split into two distinct regions around $\sin (\beta-\alpha) \sim \pm 1$. We find that in both the bbll and $\tau \tau \ell \ell$ channels, the discovery reach covers $\tan \beta$ up to about 4 , while the exclusion region extends to about 7 for $m_{A}$ up to about $600 \mathrm{GeV}$. Moreover, for $m_{H^{0}} \geq 200 \mathrm{GeV}$, this channel also allows for an exclusion reach with $Z Z Z$ final states with $0.3<|\sin (\beta-\alpha)|<1$, and $\tan \beta$ up to 4.5 for $m_{A}$ around $400 \mathrm{GeV}$. For small values of $\tan \beta$, a wide range of $m_{A}$ can be covered either by exclusion or discovery.

In the last class $g g \rightarrow H^{0} \rightarrow A Z$, we find that discovery/exclusion regions favor the negative $\sin (\beta-\alpha)$ regions, largely due to the parameter dependence of gluon fusion production $\sigma\left(g g \rightarrow H^{0}\right)$. For $m_{H^{0}}=400 \mathrm{GeV}$ and $m_{A}=50 \mathrm{GeV}$, a wide range of $\tan \beta$ versus $\sin (\beta-\alpha)$ space can be covered, except for a small stripe around $-0.15<\sin (\beta-\alpha)<$ 0.2 . For $m_{A}=200 \mathrm{GeV}$, the regions $-1 \lesssim \sin (\beta-\alpha) \lesssim-0.5$ can be excluded for all values of $\tan \beta$, while only a smaller region at low $\tan \beta$ can be discovered. For $m_{A}=50 \mathrm{GeV}$ and $\sin (\beta-\alpha)=-1$, the exclusion reach in $m_{H}$ can be as large as $450 \mathrm{GeV}$ for $\tan \beta$ around 10 , which extends even further for smaller and larger $\tan \beta$.

While extra Higgs bosons other than the observed $126 \mathrm{GeV}$ SM-like Higgs exist in many extension of the SM, the searches for those Higgses in unconventional decay channels have just started. Compared to conventional search channels of $b b, \tau \tau, W W, Z Z$ and $\gamma \gamma$, these exotic decay modes of heavier Higgses decaying into two light Higgses or one Higgs with one gauge boson can be dominant in certain regions of parameter space. In this paper, we

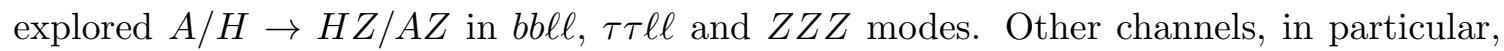
those involving charged Higgses can be very promising as well [49-53].

\section{Acknowledgments}

We thank Nathaniel Craig, Tao Han, Meenakshi Narain, Peter Loch, and John Stupak for helpful discussions. This work was supported by the Department of Energy under Grant DE-FG02-13ER41976.

Open Access. This article is distributed under the terms of the Creative Commons Attribution License (CC-BY 4.0), which permits any use, distribution and reproduction in any medium, provided the original author(s) and source are credited.

\section{References}

[1] ATLAS collaboration, Observation of a new particle in the search for the standard model Higgs boson with the ATLAS detector at the LHC, Phys. Lett. B 716 (2012) 1 [arXiv: 1207.7214] [INSPIRE].

[2] ATLAS collaboration, Combined coupling measurements of the Higgs-like boson with the ATLAS detector using up to $25 \mathrm{fb}^{-1}$ of proton-proton collision data, ATLAS-CONF-2013-034 (2013). 
[3] CMS collaboration, Observation of a new boson at a mass of $125 \mathrm{GeV}$ with the CMS experiment at the LHC, Phys. Lett. B 716 (2012) 30 [arXiv:1207.7235] [INSPIRE].

[4] CMS collaboration, Combination of standard model Higgs boson searches and measurements of the properties of the new boson with a mass near $125 \mathrm{GeV}$, CMS-PAS-HIG-13-005 (2013).

[5] ATLAS collaboration, Evidence for the spin-0 nature of the Higgs boson using ATLAS data, Phys. Lett. B 726 (2013) 120 [arXiv:1307.1432] [INSPIRE].

[6] H.P. Nilles, Supersymmetry, supergravity and particle physics, Phys. Rept. 110 (1984) 1 [INSPIRE].

[7] H.E. Haber and G.L. Kane, The search for supersymmetry: probing physics beyond the standard model, Phys. Rept. 117 (1985) 75 [INSPIRE].

[8] R. Barbieri, Looking beyond the standard model: the supersymmetric option, Riv. Nuovo Cim. 11N4 (1988) 1 [INSPIRE].

[9] J.R. Ellis, J.F. Gunion, H.E. Haber, L. Roszkowski and F. Zwirner, Higgs bosons in a nonminimal supersymmetric model, Phys. Rev. D 39 (1989) 844 [InSPIRE].

[10] M. Drees, Supersymmetric models with extended Higgs sector, Int. J. Mod. Phys. A 4 (1989) 3635 [inSPIRE].

[11] G.C. Branco et al., Theory and phenomenology of two-Higgs-doublet models, Phys. Rept. 516 (2012) 1 [arXiv:1106.0034] [INSPIRE].

[12] H.E. Haber, G.L. Kane and T. Sterling, The fermion mass scale and possible effects of Higgs bosons on experimental observables, Nucl. Phys. B 161 (1979) 493 [INSPIRE].

[13] L.J. Hall and M.B. Wise, Flavor changing Higgs-boson couplings, Nucl. Phys. B 187 (1981) 397 [inSPIRE].

[14] J.F. Donoghue and L.F. Li, Properties of charged Higgs bosons, Phys. Rev. D 19 (1979) 945 [INSPIRE].

[15] N. Craig and S. Thomas, Exclusive signals of an extended Higgs sector, JHEP 11 (2012) 083 [arXiv: 1207.4835] [INSPIRE].

[16] C.-W. Chiang and K. Yagyu, Implications of Higgs boson search data on the two-Higgs doublet models with a softly broken $Z_{2}$ symmetry, JHEP 07 (2013) 160 [arXiv:1303.0168] [INSPIRE].

[17] N.D. Christensen, T. Han, Z. Liu and S. Su, Low-mass Higgs bosons in the NMSSM and their LHC implications, JHEP 08 (2013) 019 [arXiv:1303.2113] [INSPIRE].

[18] B. Grinstein and P. Uttayarat, Carving out parameter space in type-II two Higgs doublets model, JHEP 06 (2013) 094 [Erratum ibid. 09 (2013) 110] [arXiv:1304.0028] [INSPIRE].

[19] I.M. Lewis, Closing the wedge with $300 \mathrm{fb}^{-1}$ and $3000 \mathrm{fb}^{-1}$ at the LHC: a Snowmass white paper, arXiv:1308.1742 [INSPIRE].

[20] B. Coleppa, F. Kling and S. Su, Exotic Higgs decay via AZ/HZ channel: a Snowmass whitepaper, arXiv:1308.6201 [INSPIRE].

[21] E. Brownson et al., Heavy Higgs scalars at future hadron colliders (a Snowmass whitepaper), arXiv:1308.6334 [INSPIRE].

[22] J.F. Gunion, H.E. Haber, G.L. Kane and S. Dawson, The Higgs hunter's guide, Front. Phys. 80 (2000) 1 [INSPIRE]. 
[23] B. Coleppa, F. Kling and S. Su, Constraining type II 2HDM in light of LHC Higgs searches, JHEP 01 (2014) 161 [arXiv: 1305.0002] [INSPIRE].

[24] ATLAS collaboration, Search for the neutral Higgs bosons of the minimal supersymmetric standard model in pp collisions at $\sqrt{s}=7 \mathrm{TeV}$ with the ATLAS detector, JHEP 02 (2013) 095 [arXiv: 1211.6956] [INSPIRE].

[25] CMS collaboration, Search for MSSM neutral Higgs bosons decaying to tau pairs in pp collisions, CMS-PAS-HIG-13-021 (2013).

[26] ATLAS collaboration, Discovery potential of $A / H \rightarrow \tau \tau \rightarrow \ell h$ in $A T L A S$, ATL-PHYS-PUB-2010-011 (2010).

[27] CMS collaboration, Search for a Higgs boson decaying into a b-quark pair and produced in association with b quarks in proton-proton collisions at $7 \mathrm{TeV}$, Phys. Lett. B 722 (2013) 207 [arXiv: 1302.2892] [INSPIRE].

[28] ATLAS collaboration, Search for Higgs bosons in two-Higgs-doublet models in the $H \rightarrow W W \rightarrow e \nu \mu \nu$ channel with the ATLAS detector, ATLAS-CONF-2013-027 (2013).

[29] CMS collaboration, Search for extended Higgs sectors in the $H \rightarrow$ hh and $A \rightarrow Z h$ channels in $\sqrt{s}=8 \mathrm{TeV}$ pp collisions with multileptons and photons final states, CMS-PAS-HIG-13-025 (2013).

[30] F. Febres Cordero, L. Reina and D. Wackeroth, W- and Z-boson production with a massive bottom-quark pair at the Large Hadron Collider, Phys. Rev. D 80 (2009) 034015 [arXiv:0906.1923] [INSPIRE].

[31] N. Kidonakis, Differential and total cross sections for top pair and single top production, arXiv:1205.3453 [INSPIRE].

[32] J.M. Campbell, R.K. Ellis and C. Williams, Vector boson pair production at the LHC, JHEP 07 (2011) 018 [arXiv: 1105.0020] [INSPIRE].

[33] LHC Higgs Cross Section Working Group collaboration, S. Dittmaier et al., Handbook of LHC Higgs cross sections: 1. Inclusive observables, arXiv:1101.0593 [INSPIRE].

[34] J. Alwall, M. Herquet, F. Maltoni, O. Mattelaer and T. Stelzer, MadGraph 5: going beyond, JHEP 06 (2011) 128 [arXiv:1106.0522] [INSPIRE].

[35] T. Sjöstrand, S. Mrenna and P.Z. Skands, PYTHIA 6.4 physics and manual, JHEP 05 (2006) 026 [hep-ph/0603175] [INSPIRE].

[36] DELPHES 3 collaboration, J. de Favereau et al., DELPHES 3, a modular framework for fast simulation of a generic collider experiment, JHEP 02 (2014) 057 [arXiv:1307.6346] [INSPIRE].

[37] J. Anderson et al., Snowmass energy frontier simulations, arXiv:1309.1057 [INSPIRE].

[38] ATLAS collaboration, Performance of the ATLAS muon trigger in 2011, ATLAS-CONF-2012-099 (2012).

[39] ATLAS collaboration, Performance of the ATLAS electron and photon trigger in $p-p$ collisions at $\sqrt{s}=7 \mathrm{TeV}$ in 2011, ATLAS-CONF-2012-048 (2012).

[40] LHC Higgs Cross Section Working Group collaboration, S. Heinemeyer et al., Handbook of LHC Higgs cross sections: 3. Higgs properties, arXiv:1307.1347 [INSPIRE].

[41] http://www-ekp.physik.uni-karlsruhe.de/ ott/theta/theta-auto/. 
[42] A. Avetisyan et al., Methods and results for standard model event generation at $\sqrt{s}=14 \mathrm{TeV}$, $33 \mathrm{TeV}$ and $100 \mathrm{TeV}$ proton colliders (a Snowmass whitepaper), arXiv:1308.1636 [INSPIRE].

[43] A. Avetisyan et al., Snowmass energy frontier simulations using the open science grid (a Snowmass 2013 whitepaper), arXiv:1308.0843 [INSPIRE].

[44] ATLAS collaboration, Search for the standard model Higgs boson in the decay channel $H \rightarrow Z Z^{(*)} \rightarrow 4 \ell$ with $4.8 \mathrm{fb}^{-1}$ of pp collision data at $\sqrt{s}=7 \mathrm{TeV}$ with ATLAS, Phys. Lett. B 710 (2012) 383 [arXiv:1202.1415] [InSPIRE].

[45] H.S. Cheon and S.K. Kang, Constraining parameter space in type-II two-Higgs doublet model in light of a $126 \mathrm{GeV}$ Higgs boson, JHEP 09 (2013) 085 [arXiv: 1207.1083] [INSPIRE].

[46] A. Drozd, B. Grzadkowski, J.F. Gunion and Y. Jiang, Two-Higgs-doublet models and enhanced rates for a $125 \mathrm{GeV}$ Higgs, JHEP 05 (2013) 072 [arXiv:1211.3580] [INSPIRE].

[47] S. Chang et al., Comprehensive study of two Higgs doublet model in light of the new boson with mass around $125 \mathrm{GeV}$, JHEP 05 (2013) 075 [arXiv: 1210.3439] [INSPIRE].

[48] C.-Y. Chen and S. Dawson, Exploring two Higgs doublet models through Higgs production, Phys. Rev. D 87 (2013) 055016 [arXiv: 1301.0309] [InSPIRE].

[49] T. Li and S. Su, Exotic Higgs decay via charged Higgs, in preparation.

[50] L. Basso et al., Probing the charged Higgs boson at the LHC in the CP-violating type-II 2HDM, JHEP 11 (2012) 011 [arXiv: 1205.6569] [INSPIRE].

[51] R. Dermisek, J.P. Hall, E. Lunghi and S. Shin, A new avenue to charged Higgs discovery in multi-Higgs models, JHEP 04 (2014) 140 [arXiv:1311.7208] [INSPIRE].

[52] U. Maitra, B. Mukhopadhyaya, S. Nandi, S.K. Rai and A. Shivaji, Searching for an elusive charged Higgs at the Large Hadron Collider, Phys. Rev. D 89 (2014) 055024 [arXiv: 1401.1775] [INSPIRE].

[53] B. Coleppa, F. Kling and S. Su, Charged Higgs search in $H^{ \pm} \rightarrow A W^{ \pm} / H W^{ \pm}$, in preparation.

[54] T. Hahn, S. Heinemeyer, W. Hollik, H. Rzehak and G. Weiglein, FeynHiggs 2.7, Nucl. Phys. Proc. Suppl. 205-206 (2010) 152 [arXiv:1007.0956] [INSPIRE]. 